\title{
PWR Blowdown Heat Transfer Separate-Effects Program- Thermal-Hydraulic Test Facility Experimental Data Report for Test 100
}

\author{
M. D. White \\ R. A. Hedrick
}

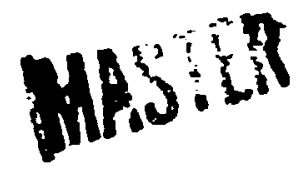

Prepared for the U.S. Nuclear Regulatory Commission Office of Nuclear Regulatory Research

Under Interagency Agreements ERDA 40-551-75 and 40-552-75 


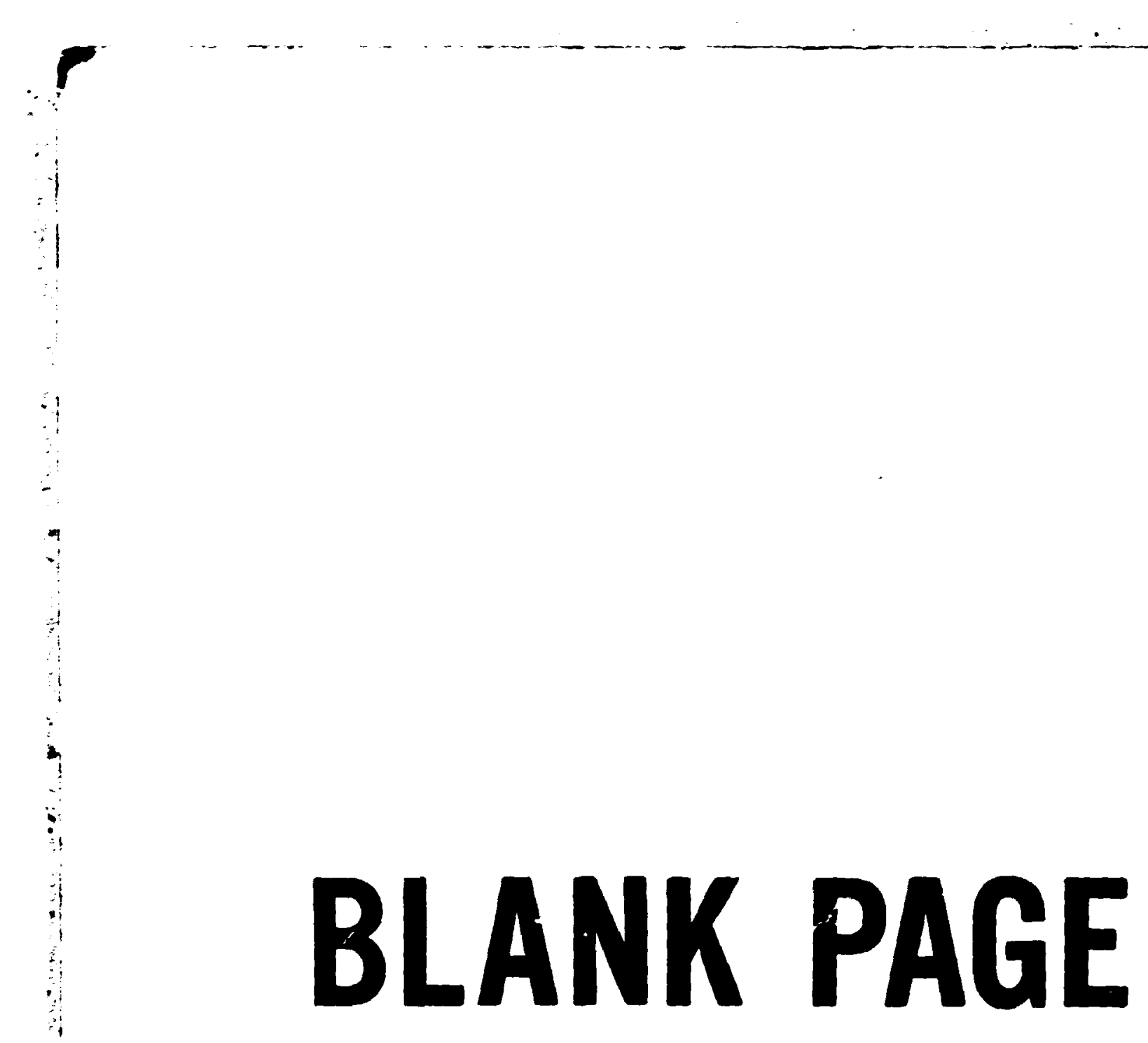




\begin{tabular}{|c|}
\hline Printed in the United States of America. Availabie from \\
National Technical Information Service \\
U.S. Depaitment of Commerce \\
5285 Port Foyal Road. Springfield. Virginia 22161 \\
Price: Printed Copy $\$ 9.75$. Microfiche $\$ 3.00$
\end{tabular}

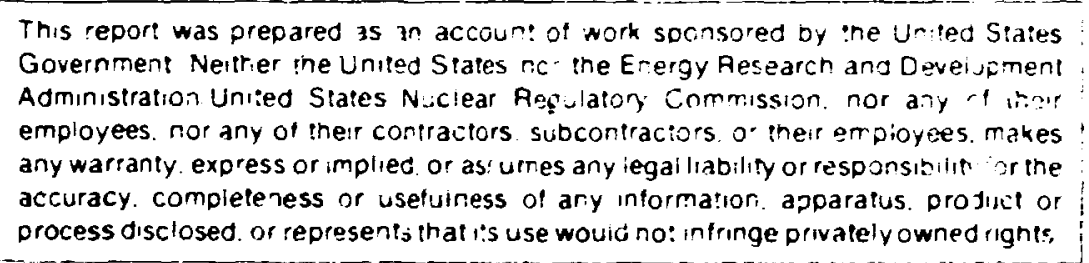


ORNL/ NUREG/TM-114

Dist. Category NRC-2

Contract W-7405-eng-26

Engineering Technoicgy Division

\begin{abstract}
PWR BLOWDOWN HEAT TRANSFER SEPARATE-EFFECIS PROGRAM - THERMAL-HYDRALLIC TEST FACILITY EXPERIMENTAL DATA REPORT FOR TEST 100
\end{abstract}

M. D. White R. A. Hedrick

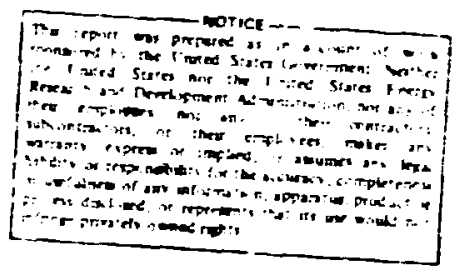

Manuscript Completed - June 22, 1977

Date Published - August :977

NOTICE: This document contains information of a preliminary nature. Tt is subject to revision or correction and therefore does aot represent a final report.

Prepared for the

U.S. Nuclear Regulatory Commission office oi Nuclear Regulatory Research

Under Interagency Agreements ERDA 40-5.51-75 and 40-552-75

Prepared by the OAK RIDGE NATIONAL LABORATORY

Oak Ridge, Tennessee 37830

operated by

UNION CARBIDE CORPORATION

for the

ENERGY RESEARCH AND DEVELOPMENT ADMINISTRATION

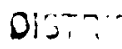


CONTENTS

FIGURES $\ldots \ldots \ldots \ldots \ldots \ldots \ldots \ldots \ldots \ldots \ldots \ldots \ldots \ldots \ldots \ldots \ldots \ldots \ldots \ldots \ldots \ldots$

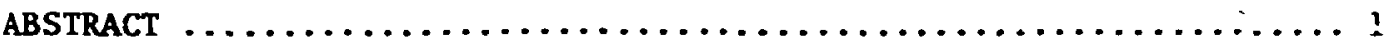

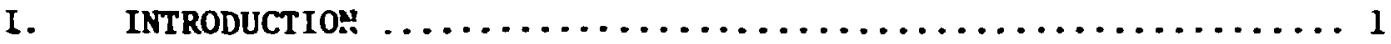

II. SYSIEM, PROCEDURES, CONDITIONS, AND EVENTS FOR TEST $100 \ldots \ldots 2$

1. Systen Corfiguration and Test Procedure ............. 2

2. Initial Test Conditions and Sequence of Events ........4

III. DATA PRESENTATION $\ldots \ldots \ldots \ldots \ldots \ldots \ldots \ldots \ldots \ldots \ldots \ldots \ldots \ldots$

\section{TABLES}

Table 1. Desired vs actual prerupture condtions $\ldots \ldots \ldots \ldots \ldots \ldots$

Table 2. Prerupture primary-coolant terperature and pressure

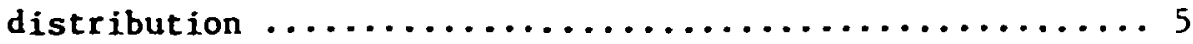

Table 3. Sequence of events during test $100 \ldots \ldots \ldots \ldots \ldots \ldots \ldots$

Table 4. Precision of experimental measurements for test $100 \ldots \ldots 7$

Table 5. Datis presentation for test $i 00 \ldots \ldots \ldots \ldots \ldots \ldots \ldots$ 


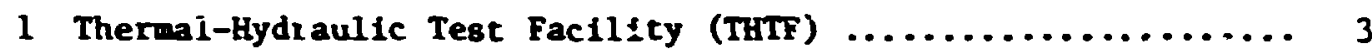

2 Identification of THIF Heater Rod and Subchannel Locations in Bundles 1 and $2 \ldots \ldots \ldots \ldots \ldots \ldots \ldots \ldots \ldots \ldots \ldots \ldots \ldots \ldots, 23$

3 THTF Loop Section Deta1ls ............................ 24

4 Tentative Location: of Therwocouples in THTr Bunile 1

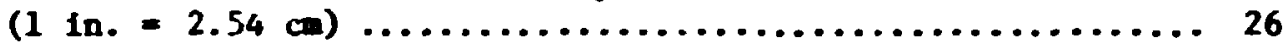

[Figures t through 504 have no page numbers assigned in Eicrofiche edition of this report. I

5 TE-30LAD, Sheath Therwocouple, Rod 1, Level D ............ 27

6 TE-304AD, St.eath Thernocouple, Rod 4, Level D ............. 27

7 TE-309AD, Sheach Thermocouple, Rod 9, Level D ........... 28

8 TE-310AD, Sheath Thermocouple, Rod 10, Level D .......... 28

9 TE-312AD, Sheath Thermocouple, Rod 12, Level D ............ 29

$10 \mathrm{TE}-313 \mathrm{AD}$, Sheath Thermocouple, Rod 13, Level D ............. 29

11 TE-317AD, Sheath Thermocouple, Rod 17, Level D ........... 30

12 TE-318AD, Sheath Thermocouple, Rod 18, Level D ........... 30

13 TE-320AD, Sheach Thermocouple, Rod 20, Level D ........... 31

14 TE-322AD, Sheath Thermocouple, Rod 22, Level D ........... 31

$15 \mathrm{TE}-323 \mathrm{AD}$, Sheath Thermocoupie, Rod 23, Level $\mathrm{D} \ldots \ldots \ldots \ldots \ldots . \ldots 32$

16 TE- $25 \mathrm{AD}$, Sheath Thermocouple, Rod 25, Level $\mathrm{D} \ldots \ldots \ldots \ldots \ldots, 32$

17 TE-326AD, Sheath Thermocouple, Rod 26, Level D ............ 33

$18 \mathrm{TE}-331 \mathrm{AD}$, Sheath Thermocouple, Rod 31, Level D ........... 33

19 TE-333AD, Sheath Thermocouple, Rod 33, Leve! D ............ 34

20 TE-338AD, Sheath Th :reocouple, Rod 38, Level $\mathrm{D} . \ldots \ldots \ldots \ldots . . . .34$

21 TE-339AD, Sheath Therwocouple, Rod 39, Level D ........... 35

22 TE-341AD, Sheach Thermocouple, Rod 41, Level D ........... 35

23 IE-349AD, Sheath Therwocouple, Rod 49, Level D ............ 36

24 TE-301AE, Sheath Thermocouple, Rod 1, Level E ............. 36

25 TE-304AE, Sheath Therwocouple, Rod 4, Level E ........... 37

26 TE-309AF, Sheath Thermocouple, Rod 9, Level E ............ 37

27 TE-31CAE, Sheath Thermocouple, Rod 10, Level E ........... 38

28 TE-312AE, Sheath Thermocoupla, Rod 12, Level E ........... 38 
Page

29 TE-313AE, Steath Therncouple, Rod 13, Level E ........... 39

30 TE-317AE, Sheath Theracouple, Rod 17, Level E .......... 39

31 TE-318AE, Sheath Thernocouple, Rod 18, Level E ...........40

32 TE-320AE, Sheath Therwocouple, Rod 20, Level E .......... 40

33 TE-322AE, Sheath Therwocouple, Rod 22, Level E .......... 41

34 TE-323AE, Sheath Thernocouple, Rod 23, Level E $\ldots \ldots \ldots \ldots \ldots 41$

35 TE-324AE, Sheath Thernocouple, Rod 24, Level E $\ldots \ldots \ldots \ldots \ldots 42$

36 TE-325AE, Sheath Therwocouple, Rod 25, Level E ......... 42

37 TE-326AE, Sheath Thermocouple, Rod 26, Level E .......... 43

38 TE-331AE, Sheath Thermocouple, Rod 31, Level E ........... 43

39 IE-333AE, Sheath Thermocouple, Rod 33, Level E ........... 44

40 TE-338AE, Sheath Thermocouple, Rod 38, Level E .......... 44

41 TE-339AE, Sheath Thernocouple, Rod 39, Level E .......... 45

42 TE-341AF, Sheath Therwocouple, Rod 41, Level E .......... 45

43 TE-349AE, Sheath Thermocouple, Rod 49, Level E ........... 46

44 TE-301BF, Sheath Thermocouple, Rod 1, Level F ........... 46

45 TE-KC.4BF, Sheath Therwocouple, Rod 4, Level F ........... 47

$46 \mathrm{TE}-3 \mathrm{Jj} 9 \mathrm{BF}$, Sheath Thermocouple, Rod 9, Level F $\ldots \ldots \ldots \ldots \ldots 47$

47 TE-310BF, Sheath Thermocouple, Rod 10, Level F $\ldots \ldots \ldots \ldots \ldots 48$

48 IE-312BF, Sheath Thermocouple, Rod 12, Level F $\ldots \ldots \ldots \ldots \ldots 48$

49 TE-313BF, Sheath Themocouple, Rod 13, Level F......... 49

$50 \mathrm{TE}-317 \mathrm{BF}$, Sheath Therwocouple, Rod 17, Level F .......... 49

$51 \mathrm{TE}-318 \mathrm{BF}$, Sheath Therwocouple, $\operatorname{Rod} 18$, Level $\mathrm{F} \ldots \ldots \ldots \ldots \ldots$ so

S2 TE-320BF, Sheath Therrocouple, Rod 20, Level $F \ldots \ldots \ldots \ldots \ldots$....... 50

$53 \mathrm{TE}-322 \mathrm{BF}$, Sheath Thermocouple, Rod 22, Level $\mathrm{F} \ldots \ldots \ldots \ldots \ldots .51$

$54 \mathrm{TE}-323 \mathrm{BF}$, Sheath Thermocouple, Rod 23, Level $\mathrm{F} \ldots \ldots \ldots \ldots \ldots 51$

55 TE-3243F, Sheath Thermocouple, Rod 24 , Level F ......... 52

56 TE-325BF, Sheath Thermocouple, Rod 25, Level F .......... 52

$57 \mathrm{TE}-326 \mathrm{BF}$, Sheath Therwocouple, Rod 26, Level F ........... S3

$58 \mathrm{TE}-331 \mathrm{BF}$, Sheath Thermocouple, Rod 31, Level F ......... 53

$59 \mathrm{TE}-333 \mathrm{BF}$, Sheath Thermocouple, Rod 33, Level F $\ldots \ldots \ldots \ldots \ldots 54$

50 TE-338BF, Sheath Thermocouple, Rod 38, Level F .......... 54

$61 \mathrm{TE}-339 \mathrm{BF}$, Sheath Thermocouple, Rod 39, Leve1 $: \ldots . \ldots \ldots \ldots \ldots$ s5

$62 \mathrm{TE}-341 \mathrm{BF}$, Sheath Thermocouple, Rod 41, Level $\mathrm{F} \ldots \ldots \ldots \ldots \ldots 5$ 
$63 \mathrm{TE}-349 \mathrm{BF}$, Sheath Thermocouple, $\operatorname{Rod} 49$, Level F $\ldots \ldots \ldots \ldots \ldots 56$

64 TE-301BG, Sheath Thermocouple, Rod 1, Level G ............ 56

65 TE-304BG, Sheath Thernocouple, Rod 4, Level G ........... 57

66 TE-309BG, Sheath The raocouple, Rod 9, Level G ........... 57

67 TE-310BG, Sheath Thermocouple, Rod 10, Level G .......... 58

68 TE-312BG, Sheath Therrac couple, Rod 12, Level G .......... 58

69 TE-313BG, Sheath Therwocouple, Rod 13, Level G ........... 59

70 TE-317BG, Sheath Thermocouple, Rod 17, Level G .......... 59

71 TE-318BG, Sheath Thermocouple, Rod 18, Lev $\geqq 1$ G.......... 60

72 TE-32NBG, Sheath Thermocouple, Rod 20, Level G ........... 60

$73 \mathrm{TE}-322 \mathrm{LG}$, Sheath Therwocouple, Rod 22, Level G ...........6. 61

74 TE-323BG, Sheath Tiermocouple, Rod 23, Level G ........... 61

$75 \mathrm{TE}-324 \mathrm{BG}$, Sheath Thermocouple, Rod 24, Level G .......... 62

$76 \mathrm{TE}-325 B G$, Sheath Therwocouple, Rod 25, Level G ........... 62

77 TE-326Br, Sheath Thermocouple, Rod 26, Level G ..........6 63

78 TE-331BG, Sheath Thermocouple, Rod 31, Level G ........... 63

79 TE-333BG, Sheath Thermocouple, Ra.i 33, Level G ........... 64

80 TE-338BG, Sheath Thermocouple, Rod 38, Level G ........... 64

81 TE-339BG, Sheath Thermocouple, Rod 39, Level G .......... 65

82 TE-341BG, Sheath Thermocouple, Rod 41, Level G ........... 65

83 TE-349BG, Sheath Thermocouple, Rod 49, Level G............ 66

$84 \mathrm{TE}-301 \mathrm{CH}$, Sheath Thermocouple, Rod 1, Level H ............ 66

85 TE-302AH, Sheach Thermocouple, Rod 2, Level H........... 67

86 TE-303AH, Sheath Thermocouple, Rod 3, Level H ............ 67

$87 \mathrm{TE}-304 \mathrm{CH}$, Sheath Thermocouple, Rod 4, Level H ........... 68

88 TE-305AH, Sheath Thermocouple, Rod 5, Level H ........... 68

89 TE-306AH, Sheach Thermocouple, Rod 6, Level H ........... 69

90 Tr.-307AH, Sheath Therwocouple, Rod 7, Level H .......... 69

91 TE-308AH, Sher th Thermocouple, Rod 8, Level H ........... 70

$92 \mathrm{TE}-309 \mathrm{CH}$, Sheath Thermocouple, Rod 9, Level H ............ 70

$93 \mathrm{TE}-310 \mathrm{CH}$, Sheath Thernocouple, Rod 10, Level H.......... 71

94 TE-311AH, Sheath Thermocouple, Rod 11, Level H.......... 71

$95 \mathrm{TE}-312 \mathrm{CH}$, Sheath Thermocouple, Rod 12, Level H .......... 72

$96 \mathrm{TE}-3 \mathrm{~L} 3 \mathrm{CH}$, Sheati Thermocouple, Rod 13, Level $\mathrm{H} \ldots \ldots \ldots \ldots . .72$ 
Page

97 TE-314AH, Sheath Therwocoup! e, Rod 14, Level H .......... 73

$98 \mathrm{TE}-315 \mathrm{AH}$, Sheath Thernocouple, Rod 15, Level H.......... 73

99 TE-316AH, Sheath Thernocouple, Rod 16, Level H ........... 74

100 TE-317CH, Sheath Therwocouple, Rod 17, Level H ........... 74

$101 \mathrm{TE}-318 \mathrm{CH}$, Sheath Therrucouple, $\operatorname{Rod} 18$, Level H .......... 75

102 TE-320CH, Sheath Therwocouple, Rod 20, Level H .......... 75

103 TE-321AH, Sheath Thermocouple, Rod 21, Level H.......... 76

$104 \mathrm{TE}-322 \mathrm{CH}$, Sheath Therwocouple, Rod 22, Level H.......... 76

$105 \mathrm{TE}-323 \mathrm{CH}$, Sheath Thermocouple, Rod 23, Level II ........... 77

$106 \mathrm{TE}-324 \mathrm{CH}$, Sneath Thermocouple, Rod 24, Level H .......... 77

$107 \mathrm{TE}-325 \mathrm{CH}$, Sheath Therwocouple, $\operatorname{Rod} 25$, Level н $\ldots \ldots \ldots \ldots \ldots 78$

$108 \mathrm{TE}-326 \mathrm{CH}$, Sheath Therwocouple, Rod 26, Level H .......... 78

109 TE-327AH, Sheath Thermocouple, Rod 27, Level H .......... 79

110 TE-328AH, Sheath Thermocouple, Rod 28, Level H ........... 79

$111 \mathrm{TE}-331 \mathrm{CH}$, Sheath Thermocouple, Rod 31, Level H.......... 80

$112 \mathrm{TE}-333 \mathrm{CH}$, Sheath Thermocouple, Rod 33, Level H .......... 80

113 TE-336AH, Sheath Thermocouple, Rod 36, Level H.......... 81

114 TE-337AH, Sheath Thermocouple, Rod 37, Level a .......... 81

115 TE-338CH, Sheath Thermocouple, Rod 38, Level H .......... 82

116 TE-339CH, Sheath Thermocouple, Rod 39, Level H ........... 82

J.17 TE-340AH, Sheath Thermocouple, Rod 40, Level H.......... 83

$118 \mathrm{TE}-341 \mathrm{CH}$, Sheath Therwocouple, Rod 41, Level H........... 83

119 TE-342AH, Sheath Therwocouple, Rod 42, Level H .......... 84

120 TE-343AH, Sheath Thermocouple, Rod 43, Level H ........... 84

121 TE-344AH, Sheath Therwocouple, Rod 44, Level H .......... 85

122 TE-345AH, Sheath Thermocouple, Rod 45, Level H.......... 85

123 TE-346AH, Sheath Thermocouple, Rod 46, Level H.......... 86

124 TE-348AH, Sheath Thermocouple, Rod 48, Level H ............ 86

125 TE-349CH, Sheach T.lermocouple, Rod 49, Level H ............ 87

$126 \mathrm{TE}-301 \mathrm{CI}$, Sheath Thermocouple, Rod 1, Level I ........... 87

127 TE-302AI, Sheath Therwocouple, Rod 2, Level I ........... 88

128 TE-303AI, Sheath Therwocouple, Rod 3, Level I ........... 88

129 TE-304CI, Sheath Thermocouple, Rod 4, Level I ............89

130 TE-305AI, Sheath Thermocouple, Rod 5, Level I ........... 89 
Page

131 TE-306A:, Sheath The rmocouple, Rod 6, Level I ........... 90

132 TE-307AI, Sheatr. Thermocouple, Rod 7, Level I ........... 90

133 TE-308AI, Sheath Thermocouple, Rod 8, Level I $\ldots \ldots \ldots \ldots \ldots .91$

134 TE-309r.I, Sheath Thermocouple, Rod 9, Level I ............ 91

135 TE-310CI, Sheath Thermocouple, Rod Ii, Level I ........... 92

136 TE-31 1AI, Sheath Thermocouple, Rod il, Level I .......... 92

$137 \mathrm{TE}-312 \mathrm{CI}$, Sheath Thermocouple, kod 12, Level I ........... 93

$138 \mathrm{TE}-313 \mathrm{CI}$, Sheath Thermocouple, Rod :3, ievel I ........... 93

139 TE-314AI, Sheath Thermocouple, Rod 14, Level I .......... 94

I40 TE-315AI, Sheath Thermocouple, Rod 15, Level I ........... 94

141 TE-316AI, Sheath Thermocouple, Rod 16, Level I ........... 95

142 TE-317CI, Sheath Thermocouple, Rod 17, Level I ........... 95

$143 \mathrm{TE}-318 \mathrm{CI}$, Sheath Thermocouple, Rod 18, Level I ........... 96

144 TE-320CI, Sheath Thermocouple, Rod 20, Level I ........... 96

145 TE-321AI, Sheath Thermocouple, Rod 21, Level I ........... 97

$146 \mathrm{TE}-322 \mathrm{CI}$, Sheath Thermocouple, Rod 22, Level I ........... 97

$147 \mathrm{TE}-323 \mathrm{CI}$, Sheath Thermocouple, Rod 23, Level I ........... 98

$148 \mathrm{TE}-324 \mathrm{CI}$, Sheath Thermocouple, Rod 24, Level I ........... 98

149 TE-325CI, Sheath Thermocouple, Rod 25, Level I ............ 99

150 TE-326CI, Sheath Thermocouple, Rod 26, Level I ........... 99

151 TE-327AI, Sheath Thermocouple, Rod 27, Level I ............ 100

152 TE-328AI, Sheath Thermocouple, Rod 28, Level I ........... 100

153 TE-331CI, Sheath Thermocouple, Rod 31, Level I ............ 101

154 TE-333CI, Sheath Thermocouple, Rod 33, Level I ............ 101

$155 \mathrm{TE}-336 \mathrm{AI}$, Sheath Thermocouple, Rod 36, Level I ........... 102

156 TE-337AI, Sheath Thermocouple, Rod 37, Level I .......... 102

$157 \mathrm{TE}-333 \mathrm{CI}$, Sheath Thermocouple, Rod 38, Level I ........... 103

$158 \mathrm{TE}-339 \mathrm{CI}$, Sheath Thermocouple, Rod 39, Level I .......... 103

159 TE-340AI, Sheath Thermoccuple, Rod 40, Level I ........... 104

$160 \mathrm{TE}-341 \mathrm{CI}$, Sheath Thermocouple, Rud 41, Level I ........... 104

$161 \mathrm{TE}-342 \mathrm{AI}$, Sheath Thermocouple, $\operatorname{Rod} 42$, Level I ........... 105

162 TE-343AI, Sheath Thermocouple, Rod 43, Level I ........... 105

$163 \mathrm{TE}-344 \mathrm{AI}$, Sheath Thermocouple, $\operatorname{Rod} 44$, Level I .......... 106

164 TE-345AI, Sheath Thermocouple, Rod 45, Level $1 \ldots . . \ldots \ldots . . .106$ 
Page

165 TE-346AI, Sheath Thermocouple, Rod 46, Level $1 \ldots \ldots \ldots \ldots . \ldots 107$

166 TE-348AI, Sheath Thermocouple, Rod 48, level I .......... 0 T

16 T TE-349CI, Sheath Thermocouple, Rod 49, Lev r.i I .......... 108

168 TF-301DJ, Sheath Thermocouple, Rod 1, Lavel J ............ 108

$169 \mathrm{TE}-302 \mathrm{CJ}$, Sheath Therwocouple, Rud 2, Level J ............ 109

170 TE-303CJ, Sheath Therwocouple, Rod 3, Level J ............. 109

171 TE-304DJ, Sheach Thermocouple, Rod 4, Level J ............ 110

172 TE-305CJ, Sheath Therwocouple, Rod 5, Level J ............. 110

173 TE-306CJ, Sheath Thermocouple, Rod 6, Level J ............ 111

174 TE-307CJ, Sheath Thermocouple, Rod 7, Level J ........... 111

175 TE-308CJ, Sheath Thermocouple, Rod 8, Level J ............ 112

176 TE-309DJ, Sheath Thenocouple, Rod 9, Level .J ............ 112

177 TE-310DJ, Sheath Theruocouple, Rod 10, Level J ...........113

$178 \mathrm{TE}-311 \mathrm{CJ}$, Sheath The roocouple, Rod 11, Level J ...........113

179 TE-312DJ, Sheath Thermocouple, Rod 12, Level J ........... 114

180 TE-313DJ, Sheath Thermocouple, Rod 13, Level J ........... 114

181 TE-314CJ, Sheath Therwocouple, Rod 14, Level J ........... 115

182 TE-315CJ, Sheath Thermocouple, Rod 15, Level J .......... 115

$183 \mathrm{TE}-316 \mathrm{CJ}$, Sheath Therwocouple, Rod 16, Leval J ........... 116

184 TE-317DJ, Sheath Thermosoupie, Rod 17, Leve1 J ......... 116

185 TE-318DJ, Sheath Thermocouple, Rod 18, Level J ........... 117

186 TE-320DJ, Sheath Therwocouple, P.od 20, Level J ........... 117

187 TE-321CJ, Shea:h Thermocouple, Rod 21, Level J ........... 118

$188 \mathrm{TE}-322 \mathrm{DJ}$, Sheath Thermocouple, Rud 22, Level J ............ 118

$189 \mathrm{TE}-323 \mathrm{DJ}$, Sheatn Thermocouple, Rod 23, Level J ........... 119

$190 \mathrm{TE}-324 \mathrm{DJ}$, Sheath Thermocouple, Rod 24, Level J ............. 119

191 TE-325DJ, Sheath Thermocouple, Rod 25, Level J ........... 120

192 TE-326DJ, Sheath Thermocouple, Rod 26, Level J ........... 120

193 TE-327CJ, Sheath Thermocouple, Rod 27, Level J ........... 121

$194 \mathrm{TE}-328 \mathrm{CJ}$, Sheath Thermocouple, Rod 28, Level J ........... 121

195 TE-331DJ, Sheath Therwocouple; Rod 31, Level J............ 122

$196 \mathrm{TE}-333 \mathrm{DJ}$, Sheath Thermocouple, Rod 33, Level J ............ 122

197 TE-336CJ, Sherth Thermocuuple, Rod 36, Level J .......... 123

$198 \mathrm{TE}-337 \mathrm{CJ}$, Sheath Thermocuuple, Rod 37, Level J ........... 1.23 
199 TE-338DJ, Sheath Thermocouple, Rod 38, Level J ........... 124

200 TE-339DJ, Sheach Therwocouple, Rod 39, Level J ........... 124

$201 \mathrm{TE}-340 \mathrm{CJ}$, Sheath Therwocouple, Rod 40, Level J ........... 125

202 TF-341DJ, Sheath Thersocouple, Rod 41, Level J ........... 125

203 TE-342CJ, Sheath Thermocouple, kod 42, Level J .......... 126

$204 \mathrm{TE}-343 \mathrm{CJ}$, Sheath Thermocouple, Rod 43, Leve? J ........... 126

$205 \mathrm{TE}-344 \mathrm{CJ}$, Sheath Thermecouple, $\operatorname{Rod} 44$, Level J .......... 127

206 TE-345CJ, Sheath Therwocouple, Rod 45, Levei J ........... 127

207 TE-346CJ, Sheath Thermocouple, Rod 46, Level J ............ 128

$208 \mathrm{TE}-347 \mathrm{CJ}$, Sheath Thernocouple, Rod 47, Level J .......... 128

$209 \mathrm{TE}-348 \mathrm{CJ}$, Sheath Thernocouple, Rod 48, Level J ........... 129

Z10 IE-349DJ, Sheach Thermocouple, Rod 49, Level J ........... 129

211 TE-301 DK, Sheath Thermocouple, Rod 1, Level K ............ 130

212 TE-304DK, Sheath Therrsocouple, Rod 4, Level K ............ 130

213 TE-309DK, Sheath Thermocouple, Rod 9, Level K ............ 131

214 TE-310DK, Sheach Thermocsuple, Rnd 10, Level K.......... 13:

$215 \mathrm{TE}-312 \mathrm{DK}$, Sheath The rmocouple, $\operatorname{Rod} 12$, Level $k \ldots \ldots \ldots \ldots \ldots 1$; 2

216 TE-313DK, Sheath Thermocouple, Rod 13, Level K ........... 132

217 TE-317DK, Sheath Thermocouple, Rod 17, Level K .......... 133

$218 \mathrm{TE}-318 \mathrm{DK}$, Sheath Thermocouple, Rod 18, Level $\mathrm{K} \ldots \ldots \ldots \ldots \ldots 133$

219 TE-320DK, Sheath Thermocouple, Rod 20, Level K ........... 134

220 TE-322DK, Sheath Thermc couple, Rod 22, wevel K .......... 134

221 TE-323DK, Sheach Therr couple, Rod 23, Level K ........... 135

222 TE-324DK, Sheath Thermocouple, Rod 24, Level K ........... 135

223 TE-325DK, Sheath Thermocouple, Rod 25, l.evel K .......... 136

224 TE-326DK, Sheath Thermocouple, $\operatorname{Rod} 26$, Level $K \ldots \ldots \ldots \ldots \ldots .36$

225 TE-331DK, Sheath Thermcouple, $\operatorname{Rod} 31$, Level $K \ldots \ldots \ldots \ldots . . .137$

226 TF,-333DK, Sheath Thermocouple, Rod 33, Level K ........... 137

227 TE-338DK, Sheath Thermocouple, Rod 38, Level K .......... 138

228 TE-339DK, Sheath Thermocouple, Rod 39, Lesel K ........... 1.38

229 TE-341DK, Sheath Thernocouple, Rod 41, Level K ........... 139

230 TE-349DK, Sheath Thermocouple, Rod 49, Levei K ........... 139

231 TE-301EL, Sheach Thermocouple, Rod 1, Level L ........... 140

232 TE-302Cl, Sheath Thermocouple, Rod 2, Level L ............ 140 
233 TE-303CL, Sneath Thermocouple, Rod 3, Level L ........... 141

234 TE-304EL, Sheath Thermocouple, Rod 4, Level L ............ 141

235 TE-305CL, Sheath Thermocouple, Rod 5, Level L ............ 142

236 TE-306CL, Sheath Thermocouple, Rod 5, Level L ............ 142

237 TE-307CL, Sheath Thermocouple, Rod 7, Level L ........... 143

$238 \mathrm{TE}-3 \mathrm{~J} 8 \mathrm{CL}$, Sheath Thermocouple, Rod 8, Level L ............ 143

239 TE-309EL, Sheath Thermocouple, Rod 9, Level L ............ 144

240 TE-310EL, Sheath Thermocouple, Rod 10, Level L ........... 144

241 TE-jilCL, Sheath Thermocouple, Rod 11, Level L ........... 145

242 TE-312EL, Sheath Thermocouple, Rod 12, Level L .......... 145

243 E-313EL, Sheath Thermocouple, Rod 13, Leve1 L ........... 146

244 TE-314CL, Sheath Thermocouple, Rod 14, Level L ........... 14.6

245 TE-315CI, Sheath Thermocouple, Rod 15, Level L ........... 147

$246 \mathrm{TE}-316 \mathrm{CL}$, Sheath Thermocouple, Ridd i6, Level L ............ 147

247 TE-317EL, Sheath Thermocouple, Risd 17, Level L .......... 148

248 TE-318EL, Sheath Thermocouple, Rod 18, Level L ........... 148

249 TE-320EL, Sheath Thermocouple, Roc 20, Level L ............ 149

250 TE-321CL, Sheath Thermocouple, Rod 21, Levil L ............ 149

251 TE-322EL, Sheath Thermocouple, Rod 22, Level I. .......... 150

252 TE-323EL, Sheath Therm couple, Rod 23. Jevel L ........... 150

$253 \mathrm{TE}-324 \mathrm{EL}$, Sheath Thermocouple, Rod 24, Level L ........... 151

$254 \mathrm{TE}-325 \mathrm{EL}$, Sheath Thermocouple, Rod 75 , Levei $\mathrm{L} \ldots \ldots \ldots \ldots \ldots 1$

255 TE-326EL, Sheath Thermocouple, Rod 26, Level L ........... 152

256 TE-327Ci, Sheath Thermocouple, Rod 27, Level L ........... 152

257 TE-328CL, Sheath Thermocouple, Rod 28, Level L ........... 153

$258 \mathrm{TE}-331 \mathrm{EL}$, Sheath Thermocouple, Rod 31, Level L .......... 153

259 TE-333EL, Sheath Thermocouple, Rod 33, Level L ........... 154

260 TE-336CL, Sheath Thermocouple, Rod 36, Level L ........... 154

$261 \mathrm{TE}-337 \mathrm{CL}$, Sheath Thermocouple, Rod 37, Level L ........... 155

262 TE-338EL, Sheath Thermocouple, Rod 38, Level L ........... 155

263 TE-339EL, Stieath Thermocouple, Rod 39, Level L ........... 156

264 TE-340CL, Sheath Thermocouple, Rod 40, Level L ........... 156

265 TE-341EL, Sheath Thenccouple, Rod 41, Level L ............57

266 TE-342CL, Sheath Thermoccuple, Rod 42, Level L .............57 
Page

267 TE-3L 3CL, Sheath The rmocouple, Rod 43, Level L .......... 158

$268 \mathrm{TE}-344 \mathrm{CL}$, Sheath Thermocouple, Rod 1.4 , Level L ............ 158

269 TE-345CL, Sheath Thermocuuple, Rod 45, Level L ........... 159

$270 \mathrm{TE}-346 \mathrm{CL}$, Sheati. Thermocouple, Rod 46, Level L ........... 159

$271 \mathrm{TE}-347 \mathrm{CL}$, Sheatt. The rmocouple, Rod 47, Level L ........... 160

272 TE-349..L. Sneath Thermocouple, Rod 48, Level L ........... 160

273 TE-349EL, Sheath Thermocouple, $\operatorname{Rod} 49$, Level I. ........... 161

274 TE-301FM, Sheath Thermocouple, Rod 1, Level M ............ 161

275 TE-304EM, Sheath Thermocouple, Rod 4, Level M ............ 162

276 TE-309EM, Sheath Thermocouple, Rod 9, Level M ............ 162

277 TE-325Ex, Sheath Thermocouple, Rod 25, Level M .......... 163

$278 \mathrm{TE}-301 \mathrm{FN}$, Sheath Thermocouple, Rod 1, Level N ............ 163

279 TE-304FN, Sheath Thermocouple, Rod 4, Level N ............ 164

280 TE-325FN, Sheath Thermocouple, Rod 25, Level $N \ldots \ldots \ldots \ldots \ldots 164$

281 TE-301F0, Sheath Thermocouple, Rod 1, Level $0 \ldots \ldots \ldots \ldots \ldots . \ldots 165$

282 TE-3C4FO, Sheath Thermocouple, Pod 4, Level $0 \ldots \ldots \ldots \ldots \ldots$

283 TE-3C9Fo, Sheath Thermocouple, $\operatorname{Rod} 9$, Level $0 \ldots \ldots \ldots \ldots \ldots 6$

284 TE-310EO, Sheath Thermocouple, Rod 10, Level $0 \ldots \ldots \ldots \ldots \ldots 166$

285 TE-312EO, Sheath Thermocouple, Rod 12, Level $0 \ldots \ldots \ldots \ldots \ldots 167$

286 TE-313EO, Sheath Thermocouple, Rod 13, Level $0 \ldots \ldots \ldots \ldots . \ldots 167$

287 TE-317EO, Shea: $h$ Thermocouple, Rod 17, Level $0 \ldots \ldots \ldots \ldots \ldots 168$

288 TE-318EO, Sheath Thermocouple, Rod 18, Level $0 \ldots \ldots \ldots \ldots \ldots 168$

289 TE-320EO, Sheath Tnermocouple, Rod 20, Level $0 \ldots \ldots \ldots \ldots . .69$

290 TE-322EO, Sheath Thermosouple, Rod 22, Le $:=10 \ldots . . . . . . .169$

291 TE-323E0, Sheath Thermocouple, Rod 23, Level $0 \ldots \ldots \ldots \ldots \ldots$.

292 TE-324EO, Sheath Thermocouple, Rod 24, Level $0 \ldots \ldots \ldots \ldots \ldots . . \ldots$

$293 \mathrm{TE}-325 \mathrm{FO}$, Sheath Thermocouple, $\operatorname{Rod} 25$, Level $0 \ldots \ldots \ldots \ldots \ldots 171$

294 TE-326EO, Sheath Thermocouple, Iod 26, Level $0 \ldots \ldots \ldots \ldots \ldots . . \ldots 1$

295 TE-331EO, Sheath Thermocouple, Rod 31, Level $0 \ldots \ldots \ldots \ldots \ldots . . .172$

296 TE-333Eo, Sheath Therwocouple, Rod 33, Level $0 \ldots \ldots \ldots \ldots \ldots 172$

297 TE-338EO, Sheath Thermocouple, Rod 38, Level $0 \ldots \ldots \ldots \ldots \ldots 173$

298 TE-339E0, Sheath Thermocouple, Rod 39, Level $0 \ldots \ldots \ldots \ldots \ldots 173$

299 TE-341E0, Sheath Thermocouple, Rod 41, Level $0 \ldots \ldots . \ldots \ldots . . .174$

$300 \mathrm{TE}-349 \mathrm{EO}$, Sheath Thermocouple, Rod 49, Level $0 \ldots \ldots \ldots \ldots \ldots . . \ldots 174$ 
301 TE-301ME, Middle Thermocoupie, Rod 1, Level E ............ 175

302 TE-318ME, Middle Thermocouple, Rod 18, Level E ........... 175

303 TE-322ME, Middle Thermocuuple, Rod 22, Level E ........... 176

304 TE-301MF, Middle Thermocouple, Rod 1, Level F .......... 176

305 TE-318M, Middle Thermocouple, Rod 18, Level F .......... 177

306 TE-322MF, Middle Thermocouple, Rod 22, Level F ........... 177

307 TE-301MG, Hiddle Thermocouple, Rod 1, Level G ............ 178

$308 \mathrm{TE}-318 M G$, Kiddie thermocouple, Rod 18, Level G ........... 178

309 TE-322MG, Middle Thermocouple, Rod 22, Level G ........... 179

310 TE-325MG, Mildle Therwocouple, Rod 25, Level G ........... 179

311 TE-318MH, Middle Thermocouple, Rod 18, Level H ........... 180

312 TE-322MFi, Middle Thermocouple, Rod 22, Level H ........... 180

313 TE-301M, Middle Thermocouple, Rod 1, Level J ............ 181

314 TE-322MJ, Middle Thermocouple, Rod 22, Level J .......... 181

315 TE-24, Thermcouple, Horizontal Inlet Spool Piece ......... 182

316 TE-172, Thermocouple, Vertical Inlet Spool Piece .......... 182

317 TE-222, Thermocouple, Vertical Outlet Spool Piece......... 183

318 TE-4C, Thermocouple, Horizontal Outlet Spool Piece ......... 183

$319 \mathrm{TE}-152$, Test Section Inlet Line Thermocouple ............. 184

320 TE-150, Test Sectiou Bottom Elange Thermocouple ........... 184

321 TE-151, Test Section Bottom Flange Thermocouple .......... 185

322 TE-152, Test Section Bottom Flange Thermocouple .......... 185

323 TE-153, Test Section $=$ sttom Flange Thermocouple .......... 186

324 TE-175, Bundle Shroud Thermocouple, .142L/LMAX .......... 186

325 TE-176, Bundle Shroud Thermocouple, .142L/LMAX .......... 187

326 TE-177, Bundle Shroud Thermocouple, .142L/LMAX ........... 187

327 TE-178, Bundle Shroud Thermocouple, .142L/iMAX .......... 188

328 TE-179, Bundle Slıroud Thermocouple, .388L, LMAX .......... 188

329 TE-180, Bundle Shroud Thermocouple, .388L/LMAX ........... 189

$330 \mathrm{TE}-181$, Bundle Shroud Thermocouple, .388L/LMAX ............ 189

331 TE-182, Bundle Shroud Thermocouple, .388L/LMAX ............ 190

332 TE-183, Bundle Shroud Therwocouple, .633L/LMAX ........... 190

$333 \mathrm{TE}-184$, Bundle Shroud Thermocouple, .633L/LMAX ........... 191

$334 \mathrm{TE}-185$, Bundle Shroud Thermocouple, .633L/LMAX ............ 191 
$335 \mathrm{TE}-186$, Bundle Shroud Thermocouple, .633L/LAAX ........... 192

$336 \mathrm{TE}-187$, Bundle Shroud Thermocouple, .875L/LMax ............ 192

337 TE-188, Bundle Shroud Thermocuuple, .8751./LMax ........... 193

$338 \mathrm{TE}-189$, Bundle Sixoud Therwocouple, .875L/LMAX ............ 193

339 TE-190, Bundle Shroud Therwocouple, .875L/LMAX ............ 194

$340 \mathrm{TE}-212$, Test Section Outlet Line Thermocouple ............ 194

$341 \mathrm{TE}-30 \mathrm{~B}$, Heat Exchanger A Outlet Thermocouple ............. 195

342 TE-32B, Heat Exchanger B Out let Thermocnuple ............. 195

$343 \mathrm{TE}-34 \mathrm{~B}$, Heat Erchanger C Out let Thermocouple ............. 196

344 TE-520B, RBT 4 in. Jeminerailzed Hater Header Thermocouple ... 196

345 TE-5B, Heat Exchanger D Out let Thermocouple .............. 197

346 iE-1, Pressurizer Tank Top Thermocouple ................. 197

347 TE-2, Pressurizer Tank Exit Thermocouple ............... 198

348 TE-29, Inlet Blowdown Plenum Thermocouple ................ 198

349 TE-45, Outlet Blowdori Plenum Thermocouple ................ 199

350 TE-4B, Base Primary Steady State Temperature, RTD .......... 199

351 FE-620, HX B Secondary Cooling Water Flow .............. 200

352 FE-720, HX C Secondary Cooling water Flow ............. 200

353 FE-550, HX D Secondary Cocling Water Flow ............. 201

354 FE-1A, Primary Side Pump flow ....................... 201

355 FE-19, Turbine Flow Meter, Horizontal Inlet Spori Piece ...... 202

356 FE-166, Turbine Flow Meter, Vertical Inlet Spool Piece ...... 202

357 FE-216, Turbine Flow Meter, Verticai Outlet jpool Plece ...... 203

358 FE-34, Turbine Flow Meter, Horizontal Outlet Spool Piece ..... 203

359 PE-26, Transient Pressure, Horizontal Inlet Spuol Plece ...... 204

360 PE-174, Transient Pressure, Vertical Inlet Spool Piece ...... 204

361 PE-156, Test Section Inlet Plenum Transient Pressure ........ 205

362 PE-201, Test Section Outlet Plenum Transient Pressure ....... 205

363 PE-224, Transient Presgur?, Vertical Outler. Spool Pfece ...... 206

364 PE-42, Transfent Pressure, Horizontal Outiet Spool Pfece ..... 206

365 PE-44, Upstream hiain HX Transient Yressure .............. 207

366 PE-76, Primary Pump Suction Transient Pressure ........... 207

367 PT-102, Pressurizer Steady State Pressure .............. 208

368 PE-106, Pressurizer Vapor Transient Pressure .............. 208 
359 PE-412, Pressure Suppression Receiver Transient Pressure ..... 209

370 PDE-167, Transient DP, :ertical Inlet Spool Piece ............ 209

371 TE-615, Demizeralized Hater 6 in. Header Steady State Temp. .. 210

372 PDT-i99, Test Section Shroud Box Steady State UP ........... 210

373 PDE-200, Test Bundle Transient DP ................... 211

374 PDE-35, Transient DP, Horizontal Ont let Spool Piece ......... 211

375 PDE-21i, Transient DP, Vertical Outlet Spool Piece ......... 212

376 TE-627, HX B Secondary Discharge Steady State Temp., RTD ..... 212

377 PDT -48 , Main HX steady State DP .................... 213

378 PDE-78, P. imary Punp Transient DP ................... 213

379 PE-425, PSS Inlet Blowdown Line Transient Pressure ......... 214

380 PE-427, PSS Outlet Blowdown Line Transient Pressure ......... 214

$381 \mathrm{TE}-727$, HXX C Secondazy Discharge Steady State Tem., RT . ... 215

382 TE-28B, Main HX Mixing Tee Steady State Temp., RTD ......... 215

383 PDE-46, Main HX Bypass irausient DP ................. 216

384 FMFE-22, Drag Disk, Horiz.rtal Inlet Spool Piece .......... 216

385 mFE-170, Drag Disk, Vertical Inlet Spool Piece ........... 217

386 FMFE-220, Drag Disix, Verticai Outlet Spool Piere .......... 217

387 FMFE-38, Drag Disk, Horizontal Outlet Spool Piece .......... 218

388 PE-526, HX A Secondary Inlet Pressure ................ 218

389 PDE-21, Transient DP, Horizontal Inlet Spool Piece ........ 219

390 PE-16, Downstream HCV-2 Transient Pressure .............. 219

391 PE-27, Test Section Inlet Plenum Bottrom Transient Pressure ... 220

392 PE-43, Test Section Outlet Plenum Pottom Transient Pressure .. 2:0

393 PDT-30, Test Section Steady State UP ................. 221

394 PT-32, Test Section Outlet Steady State Pressure ........... 221

395 SE-72, Prinary Pump Speed ....................... 222

396 XY-4000I, Breakwire Detector, Inlet Break ............... 222

397 Xy-40000, Breakwite Detector, Outlet Break ............. 223

398 LT-100, Steady State Pressurizer Liquid Level ............ 223

399 FE-522, HX A Secondary Cooling Water Flow .............. 224

400 TE-557, HX D Secondary Discharge Steady-State Teap., RTD ..... 224

401 TE-525, aX A Secondary Discharge Steady-State Temp., RTD ..... 225

402 TE-210A, Test Section Dutlet Line Steady-State Temp., RTD .... 225 
$\boldsymbol{\pi v i 1}$

Page

403 EIM-1001B, RTD Power Supply Current ................. 226

404 ElE-11S6, Rod 1 Heater Current ...................... 226

405 EIE-12S6, Rod 2 Heater Current ...................... 227

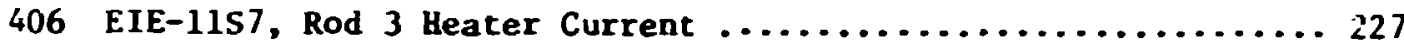

407 EIE-12S7, Rod 4 Heater Surzent ................... 228

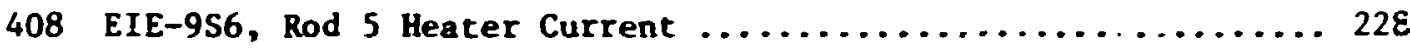

409 EIE-9S8, Rod 6 Heater Current ......................... 229

410 EIE-9S5, Rod 7 Heater Current ......................... 229

411 EIE-11S5, Rod 8 Heater Current ...................... 230

412 EIE-11S8, Rod 9 Heater Current .................... 230

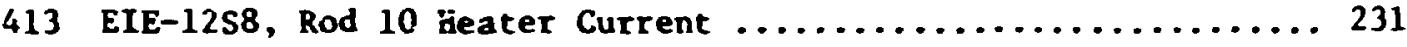

414 EIE-9S7, Rod 11 heater Current $\ldots \ldots \ldots \ldots \ldots \ldots \ldots \ldots \ldots \ldots \ldots \ldots$

415 EIE-9S4, Rod 12 Heater Current $\ldots \ldots \ldots \ldots \ldots \ldots \ldots \ldots \ldots \ldots \ldots \ldots \ldots$

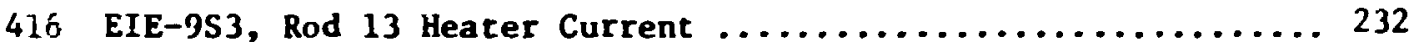

417 EIE-9S11, Rod 14 Heater Current .................... 233

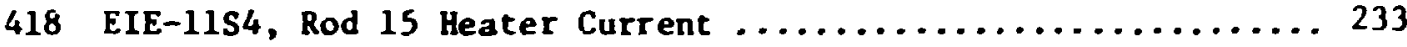

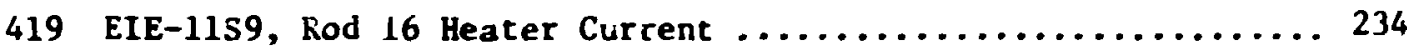

420 EIE-12S5, Rod 17 Heater Current ...................... 234

421 EIE-9S9, Rod 18 Heater Current $\ldots \ldots \ldots \ldots \ldots \ldots \ldots \ldots \ldots \ldots . \ldots . \ldots . \ldots$

422 EIE-9S10, Rod 19 Heater Current .................... 235

423 EIE-GS2, Rod 20 Heater Current .................... 236

424 EIE-9S12, Rod 21 Heater Current .................... 236

425 EIE-11S11, Rod 22 Heacer r.urrent .................... 237

426 EIE-11S10, Rod 23 Heater Current .................. 237

427 EIE-1 1S3, Rod 24 Heater Current ................... 238

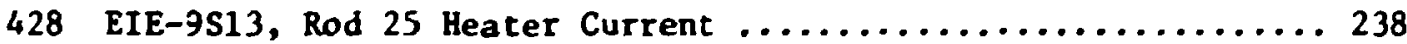

429 EIE-10S6, Rod 26 Heater Current ..................... 239

430 EIE-9S1, Rod 27 Heater Current ...................... 239

431 EIE-10S7, Rod 28 Heater Current .................... 240

432 EIE-11S2, Rod 29 Heater Current ................... 240

433 EIE-11s12, Rod 30 Heater Current $\ldots \ldots \ldots \ldots \ldots \ldots \ldots \ldots \ldots . \ldots \ldots$

434 EIE-12S2, Rod 31 Heater Current .................... 241

435 EIE-12S1, Rod 32 Heater Current ..................... 242

436 EIE-10S12, Rod 33 Heater Current .................. 242 
Page

437 EIE-10S5, Rod 34 Heater Current .................... 243

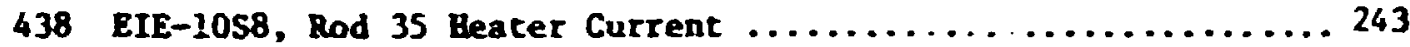

439 EIs-11S1, Rod 36 Heater Current ................... 244

440 EIE-12S4, hod 37 Heater Current .................... 244

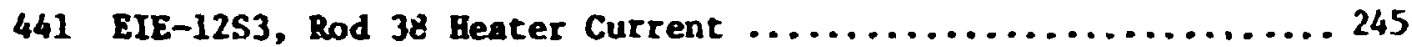

442 EIE-12S12, Rod 39 Heater Current ..................... 245

443 EIE-10\$11, Rod 40 Beater Current $\ldots \ldots \ldots \ldots \ldots \ldots \ldots \ldots \ldots \ldots . \ldots \ldots$

444 EIE-10S10, Rod $\& 1$ Heater Current $\ldots \ldots \ldots \ldots \ldots \ldots \ldots \ldots \ldots \ldots$

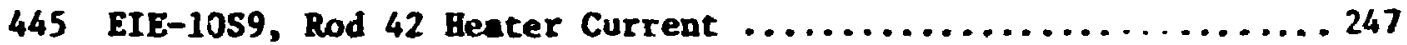

446 EIE-12S9, Rod 43 Heater Current .................... 247

447 EIE-12S10, Rod 44 Heater Current ................... 248

448 EIE-12S11, Rod 45 Heater Current $\ldots \ldots \ldots \ldots \ldots \ldots \ldots \ldots \ldots . \ldots . \ldots 248$

449 EIE-1C31, Rod 46 Heater Current .......................... 249

450 EIE-10S2, Rod 47 Heater Current ..................... 249

451 EIE-10S3, Rod 48 Heater Current ........................ 250

452 EIE-10S4, Rod 49 Heater Current .......................... 250

453 EIE-9, Generator 9 Current ............................. 251

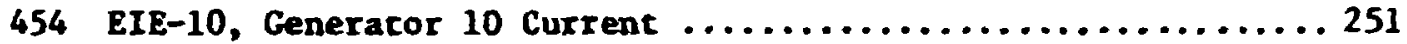

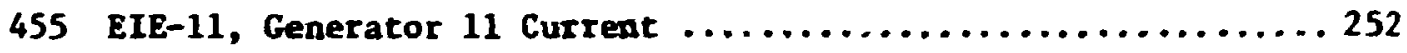

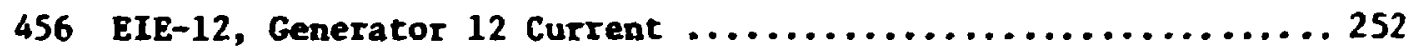

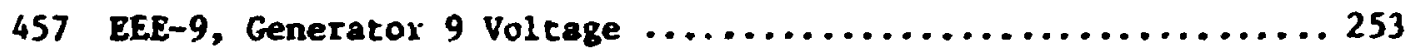

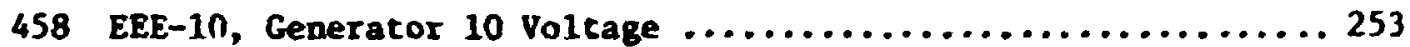

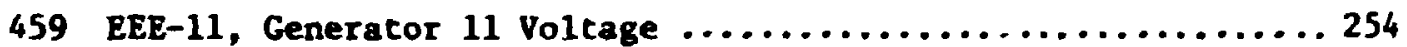

460 EEE-12, Gencrator 12 Voltage ........................ 254

$461 \mathrm{DE}-20$, Gama Densfrometer, Horizontal Inlet Spool Piece ..... 255

462 DE-168, Gama Densfiometer, Vertical Inlet Spool Plece ...... 255

463 DE-218, Gama Densitometer, Vertical Ourlet Spool Piece ...... 256

464 DE-16, Gega Deneltometer, Horizontal Outlet Spool Plece ..... 256

465 Zero Cal1bration Input, Channels $0-127 \ldots \ldots \ldots \ldots \ldots \ldots \ldots . \ldots 257$

466 Zero Calfbration Input, Channels 128-255 ............... 257

467 Zero Callbration Input, Channele 256-383 ............... 258

468 Zero Cal1bration Input, Channels $384-511 \ldots \ldots \ldots \ldots \ldots \ldots \ldots . . \ldots 258$

469 Pull-Scale Cal1bration Input, Chantels 0-127 ............. 259

470 Full-Scale ral1bration Input, Channels 128-255 ........... 259 
Page

471 Full-Scale Calibration Input, Chan els 256-383 ........... 260

472 Full-Scale Calibration Input, Channels 384-511 ........... 260

473 TE-S1, Subchannel 1 Therwocouple .................... 261

474 TE-S2, Subchannel 2 Thermocouple .................... 261

475 TE-S3, Subchannel 3 Thermocouple .................... 262

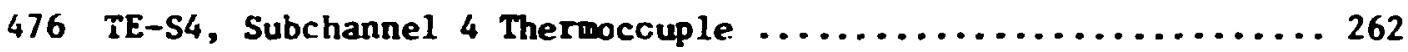

477 TE-S5, Subchannel 5 Therwoccuple .................... 263

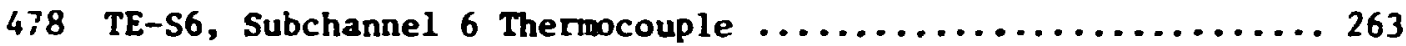

479 TE-S7, Subchannel 7 Thermocociple .................... 264

480 TE-S8, Subchannel 8 Thermocouple ................... 264

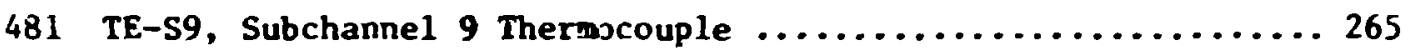

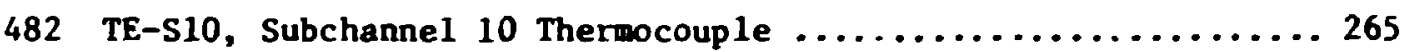

483 TE-S11, Subchannel 11 Thermocouple .................. 266

484 TE-S13, Subchannel 13 Thermocouple ................... 266

$485 \mathrm{TE}-\mathrm{S17}$, Subchannel 17 The nocouple ................. 267

486 TE-S18, Subchannel 18 Thermocouple ................. 207

487 TE-S19, Subchannel 19 Thermocouple .................. 268

488 TE-S20, Subchanne1 20 Thermoroup le .................. 268

489 TE-S25, Subchannel 25 Thermocouple .................. 269

490 TE-S26, Subchannı1 26 Thermccouple .................. 269

491 TE-S27, Subchannel 27 Thermocouple ................. 270

492 TE-S28, Subchan 1el 28 Thermocouple ................ 270

493 TE-S29, Subchannel 29 Thermocouple .................. 271

494 TE-S30, Subchanne?. 30 Thermocouple .................. 271

$495 \mathrm{TE}-\mathrm{S31}$, Subchannel 31 Therwocouple ................. 272

$496 \mathrm{TE}-\mathrm{S} 32$, Subchannel 32 Thermocouple ................. 272

497 TE-S33, Subchanne? 33 Thermocouple .................. 273

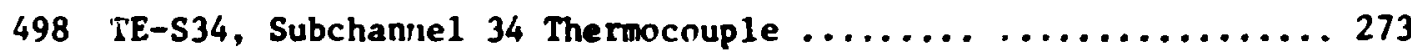

$499 \mathrm{TE}-\mathrm{S} 35$, Subchanr.el 35 Therwocouple .................. 274

$500 \mathrm{TE}-\mathrm{S} 36$, Subch inel 36 Thermocouple .................. 274

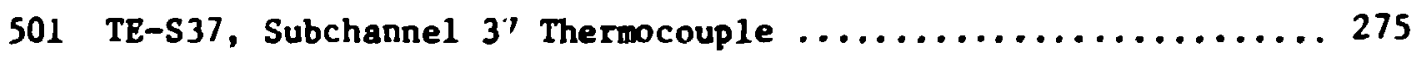

S02 TE-S46, Subchannel 46 Tharmocouple .................. 275

$573 \mathrm{TE}-555$, Subchannel 55 Thermocouple ................... 276

$504 \mathrm{TE}-\mathrm{S64}$, Subchannel 54 The rmocouple .................. 276 
, 


\title{
PWR BLOWDOWN HEAT TRANSFER SEPARATE-EFFECTS \\ PROGRAM - THERMAL-HYDRAUL IC TEST FACILITY \\ EXPERIMENTAL DATA REPORT FOR TEST 100
}

\author{
M. D. White R. A. Hedrick
}

\begin{abstract}
Reduced instrument responses ate presented for ThernalHydraulic Test Facility (THT) test 100, which is part of the ORML Pressurized-Hater-Reactor (IWR) Blowdown Heat Transfer Separate-Effects Prograe. The objective of the program is to investigate the thermal-hydraulic phenomenon governing the energy transfer and transport processes that occur during a loss-of-coolant accident in a PWR systen.

Test 100 was conducted to investigate the response of heater rod bundle 1 and instrumenced spool pieces with flow homogenizing screens to a double-ended rupture with equal break areas at the test sectior. inlet and outlet.

The primary purpose of this report is to make the reduced instrument responses during test 100 avallable. The responses are presented in graphical form in engincering units and have been analyzed only to the extent necessary to assure reasonableness and consistency.
\end{abstract}

\section{INTRODUCTION}

The Oak Rióge National Laboratory Pressurized-Water Reactor (ORNL-PPR) Blowdown Heat Transfer Program is a separate-effects study of the relations among the princtpal variables that can alter the rate of blowdown, the presence of flow reversal and rereversal, tine delay to critical heat flux, the rate at wich dryout progresses, and simflar time- and space-related functions that are lmportant in loss-of-coolant accident (LOCA) analyses. Primary test results are obtained from the Thermal-Hydraulic Test Fac1lity (THTF), a large nonnuclear fressurized-water loop incorporating a 49-rod eltetrically heaced bundle in $7 \times 7$ geometry.

THTF test 100 (conducted Apr. 23, 1976) was the first test conducted in the facility with bundle 1 in place. This test was performed to investigate the response of bundle 1 and instrumented spool pieces wth flow homogenizing screens to a double-ended rupture with equal break areas at 
the test section inlet and outlet. This was an isothermal test to provide baseline informatior: on the experimental factlity response.

The purpose of this report is to piovide the reduced instrument responses during test 100 in a readily usat? ${ }^{2}$ form to the nuclear comuntty In advance of detalled analyses and interprztztions. These data are presentizd on aicrofiche attached to the back cover of the report. Final anaiyses and interpretations are scheduled for publication six months arter the completion of test series 100 . The progrim and the experimental facilities are described in Ref. 1.

\section{SYSTEY, PROCEDURES, CONDITIONS, AND EVENTS FOR TEST 100}

\section{System Configuration and Test Procedures}

The Theral-Hydraulic Test Facilfty (THTF), shom in Fig. 1, consis:s of a test section wth a 49-rod, 3.66-m-long (12-ft) electrically heated core; a circulation loop comprised of three parallel heat exchangers with bypass, a pressurizer, a pump with bypass, and associated control valves; two rupture assemblies; and a pressure-suppression system. For test 100 a total break area of $12.54 \mathrm{~cm}^{2}\left(0.0135 \mathrm{ft}^{2}\right)$ was divided equally between the test section inlet and outlet ruptire assemblies. The THTF experimental system is described further in Ref. 1.

Since test 100 was an isothermal test, no power was applied to the electric core. The main heat exchangf:rs were drained, and the drains and vents were $1: 2 \mathrm{ft}$ open.

in preparation for the test, the loop was filled with detineralized water and the system pressure checked. Instrumentation and data acquis1tion checks were performed. During the warmup, data were taken for use in flow and pressure callbrations.

During the test, the THTF was successfully subjected to a druble-ended pipe break through the two rupture assemblies containing the orifice

1. Project Description: ORNL PWR Blowdown Heat Transfer SeparateEffects Progran - Thermal-Hydraulic Test Pacility (THTR), ORNL/NUREG/TM-2 (February 1976). 


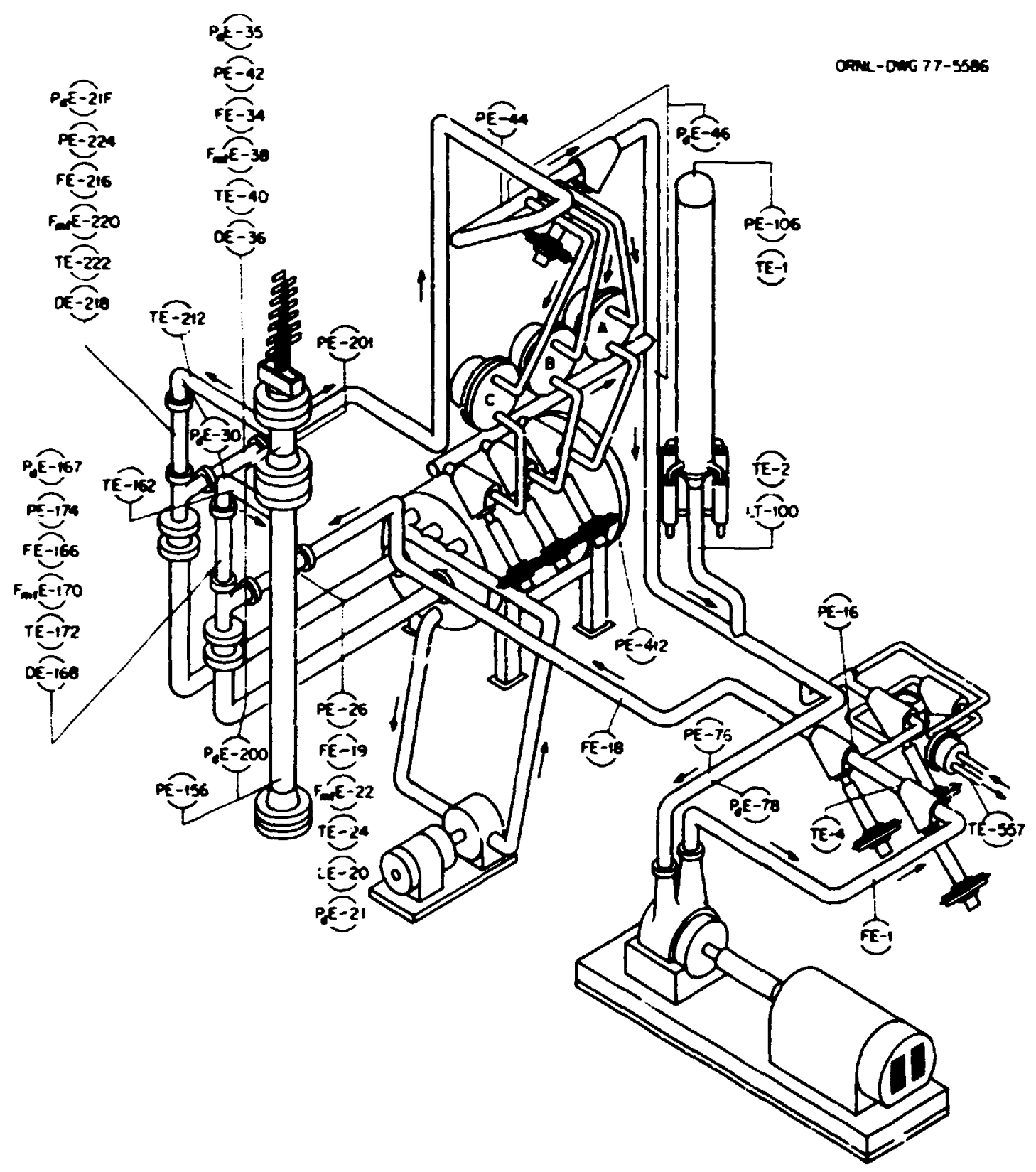

F18. 1. Thermal-Hydraulic Test Fac1lity (THTF). 
piates. The effluent from the primary system was ejected into the pressure-suppression system, which was maintained at atmospheric pressure. The primary-coolant pum was tripped coircident with break initiation.

\section{Initial Test Conditions and Sequence of E:ents}

The THTF conditions imedlately preceding rupture are given in Tables 1 and 2. The sequence of events relative to the rupture is given in Tabie 3.

Table 1. Desired us accual prerupcure conditions

\begin{tabular}{|c|c|c|c|}
\hline Paraneters & Instrument & Desired: & A.twal \\
\hline $\begin{array}{l}\text { Sysceapressure } \\
\mathrm{mN} / \mathrm{m}^{2} \\
\text { psiz }\end{array}$ & PE-20I & $\begin{array}{l}15.513 \\
2250\end{array}$ & $\begin{array}{l}15.40\} \\
22 \times 3\end{array}$ \\
\hline Core power & $\begin{array}{l}\text { EIE-9, EIE-10, EIE-11, EIE-12 } \\
\text { EEE-9. EEE-10, EFE-11, EEE-12 }\end{array}$ & zero & Zero \\
\hline $\begin{array}{l}\text { Care volumetric flow rate } \\
\mathrm{m}^{3} / \mathrm{s} \\
\mathrm{ppo}\end{array}$ & FE-19 & $\begin{array}{l}0.0265 \\
420\end{array}$ & $\begin{array}{l}7.0268 \\
\rightarrow 23\end{array}$ \\
\hline $\begin{array}{l}\text { Test section inlet cemperature } \\
\text { ok } \\
\text { - F }\end{array}$ & $T E-162$ & $\begin{array}{l}559.1 \\
547\end{array}$ & $\begin{array}{l}\text { ino. } 0.9 \\
\text { in }\end{array}$ \\
\hline $\begin{array}{l}\text { Test section outlet temperature } \\
{ }^{\circ} \mathrm{K} \\
{ }^{\circ} \mathrm{F}\end{array}$ & TE-212 & $\begin{array}{l}359.3 \\
547\end{array}$ & $\begin{array}{l}3 ; 9.9 \\
3 \rightarrow 8\end{array}$ \\
\hline \multicolumn{4}{|l|}{ Pressur izer } \\
\hline $\begin{array}{l}\text { Presssure } \\
\mathrm{mN} / \mathrm{m}^{2} \\
\text { psig }\end{array}$ & $P E-106$ & $\begin{array}{l}1: .720 \\
2: 35\end{array}$ & $\begin{array}{l}1 \div .851 \\
215 \div\end{array}$ \\
\hline $\begin{array}{l}\text { Lass liquid wacer } \\
K_{8} \\
\text { Ib }\end{array}$ & & $\begin{array}{l}31.26 \\
113\end{array}$ & $\begin{array}{l}76.48 \\
159.5\end{array}$ \\
\hline \multicolumn{4}{|l|}{ Coolant pupp } \\
\hline $\begin{array}{l}\text { Speed } \\
\text { rps } \\
\text { rpm }\end{array}$ & $S E-72$ & $\begin{array}{l}39.67 \\
3580\end{array}$ & $\begin{array}{l}60.38 \\
36 \geq 3\end{array}$ \\
\hline $\begin{array}{l}\text { Pressure different }(a) \\
\text { nN/m } \\
\text { psid }\end{array}$ & $P_{d} E-78$ & $\begin{array}{l}4.487 \\
64 i\end{array}$ & $\begin{array}{l}\text { ing } \\
65\end{array}$ \\
\hline $\begin{array}{l}\text { Pressure between } \mathrm{HCV}-2 \text { and } \mathrm{FCV}-18 \\
\mathrm{MN} / \mathrm{m}^{2} \\
\mathrm{psig}\end{array}$ & $P E-16$ & $\begin{array}{l}16.547 \\
2400\end{array}$ & $\begin{array}{l}17.361 \\
2518\end{array}$ \\
\hline
\end{tabular}


Table 2. Prexupture prienry-coolant temperature and pressure distributiona

\begin{tabular}{|c|c|c|c|c|}
\hline Location & Instrunent & $\begin{array}{c}\text { Temperature } \\
\left.\left.\right|^{\bullet K}\left({ }^{\bullet} \mathrm{F}\right)\right]\end{array}$ & $\begin{array}{l}\text { Pres } \\
{\left[\mathrm{mx} / \mathrm{m}^{2}\right.}\end{array}$ & $\begin{array}{l}\text { ssure } \\
(p s i g))\end{array}$ \\
\hline Vertical inlet spool piece & TE-172 & $560 .:(569)$ & & \\
\hline Vertical ialet spool piece & $P E-176$ & & 15.802 & $(2292)$ \\
\hline Test section inler & $\pi E-162$ & $560.9(550)$ & & \\
\hline Loner pleave & TE-150 & $559.3(547)$ & & \\
\hline Louver pleave & $P E-156$ & & 15.741 & (2283) \\
\hline Vpp::= plenu & PE-201 & & 15.603 & $(2263)$ \\
\hline Test section outlet & $\pi E-212$ & $5: 9.8(548)$ & & \\
\hline Vertical outlec spool piece & TE-222 & $559.8(548)$ & & \\
\hline Vertical outlet spool piece & $P E-226$ & & 15.534 & (2253) \\
\hline Heat exchanger inlec header & $P E-46$ & & $15 .+30$ & (2235) \\
\hline Mixed mean cemperacure & & & & \\
\hline Downst rean heat excharyers & TE-288 & $561.5(551)$ & & \\
\hline Pressurtizer surge line & TE-2 & $612.0(6+2)$ & & \\
\hline Pressurtizer & PF-106 & & 14.851 & $(2156)$ \\
\hline Primary puep suction & PE-76 & & 14.920 & $(2164)$ \\
\hline $\begin{array}{l}\text { Between anin concrol values } \\
\text { HCV-2 and FCV-19 }\end{array}$ & TE- -68 & $357.6(54 \%)$ & & \\
\hline $\begin{array}{l}\text { Between aín eancrol values } \\
\text { HCV-2 and FCV-18 }\end{array}$ & PE-16 & & 17.361 & $(2518)$ \\
\hline
\end{tabular}

Table 3. Sequence of events during test $100^{c}$

\begin{tabular}{lc}
\hline \multicolumn{1}{c}{ Event } & $\begin{array}{c}\text { Time relative } \\
\text { co rupture } \\
\text { (sec) }\end{array}$ \\
\hline Core power level established & N.A. \\
Core temperacure rise established & N.A. \\
Analog tapes and ccons fast scan started & $-1 S$ \\
Bloudoum iniclaced & 0 \\
Punp power tripped & 0 \\
Heat exchanger secondary valven closed & N.A. \\
Core power tripped & N.A. \\
\hline
\end{tabular}

$a_{\text {H.A. }}=$ noc applicable. 
III. DATA PRESENTATION

Thu recorded : nstrument responses for THTF test 100 have been processed only to the extent $\mathrm{n}_{1}$ sssary to obtain appropriate engineering units and to assure reasonableness and consistency. In converting instrument responses to engineering units, a homogeneous fluid has been assumed. Therefore, interpretation or analysis of the data must conside: the fact that the instruments way have been subjected to nonhomogeneous fluid conditions during the transient.

The reduced Instrument responses presented in this report were recorded by a computer-controlled digital data acquisition system (DAS). Further Inforation on this system may be found in Ref. 1 .

Figures 2 through 4 provide supportive information for the instrument responses (on microfiche) and indicate the reiative locations of the detectors in the THTF. Table 4 gives the precision of the recorded instrument responses, and Table 5 groups the measurements by location and provides brief coments regarding the detectors and the recorded responses. Time zero on all graphs is the time of break initiation. 
Table 4. Precision of experimental measurements for test 100

\begin{tabular}{|c|c|}
\hline System & Standard deviation \\
\hline \multicolumn{2}{|l|}{ Pressure measurement, $\mathrm{MN} / \mathrm{m}^{2}$ (psig) } \\
\hline $\begin{array}{l}\text { CCDAS } \\
\text { Analog tape system }\end{array}$ & $\begin{array}{ll}0.185 & (26.8) \\
0.197 & (28.5)\end{array}$ \\
\hline \multicolumn{2}{|l|}{ Pressure difference measurement, MN/m ${ }^{2}$ (psid) } \\
\hline $\begin{array}{l}\text { CCDAS } \\
\begin{array}{ll}6.89-\mathrm{NN} / \mathrm{m}^{2} & (1000-\text { psid) span } \\
1.38-\mathrm{NN} / \mathrm{m}^{2} & (200-p s i d) \text { span } \\
0.34-\mathrm{NN} / \mathrm{m}^{2} & \text { (S0-psid) span }\end{array}\end{array}$ & $\begin{array}{ll}0.025 & (3.6) \\
0.005 & (0.72) \\
0.001 & (0.18)\end{array}$ \\
\hline $\begin{array}{l}\text { Analog tape system } \\
6.89-\mathrm{MN} / \mathrm{m}^{2}(1200-p s i d) \text { span } \\
1.38-\mathrm{MN} / \mathrm{m}^{2}(200-p s i d) \text { span } \\
0.34-\mathrm{MN} / \mathrm{m}^{2}(50-p s i d) \text { span }\end{array}$ & $\begin{array}{ll}0.033 & (4.8) \\
0.007 & (0.95) \\
0.002 & (0.24)\end{array}$ \\
\hline Temperature measurement, ${ }^{\circ} \mathrm{K}\left({ }^{\circ} \mathrm{F}\right)$ & $2.4(4.3)$ \\
\hline \multicolumn{2}{|l|}{ Flow measurement, $\mathrm{m}^{3} / \mathrm{sec}(\mathrm{gpm})$} \\
\hline $\begin{array}{l}\text { FE-19 } \\
\text { Forward } \\
\text { Reverse }\end{array}$ & $\begin{array}{l}+0.0009-0.0012 \\
(+13.97-2.90) \\
+0.0011-0.0004 \\
(+16.77-5.70)\end{array}$ \\
\hline \multicolumn{2}{|l|}{ FE-166 } \\
\hline $\begin{array}{l}\text { Forward } \\
\text { Reverse }\end{array}$ & $\begin{array}{l}+c .0012-0.0005 \\
(+18.14-7.68) \\
+0.0010-0.0003 \\
(+16.15-5.09)\end{array}$ \\
\hline \multicolumn{2}{|l|}{$F F-216$} \\
\hline $\begin{array}{l}\text { Forward } \\
\text { Reverse }\end{array}$ & $\begin{array}{l}+0.0048-0.0041 \\
(+75.59-64.52) \\
+0.0020-0.0013 \\
(+31.79-20.72)\end{array}$ \\
\hline \multicolumn{2}{|l|}{ FE-34 } \\
\hline $\begin{array}{l}\text { Forward } \\
\text { Reverse }\end{array}$ & $\begin{array}{l}+0.0021-0.0007 \\
(+33.39-11.26) \\
+0.0124-0.0110 \\
(+197.11-174.87)\end{array}$ \\
\hline \multicolumn{2}{|l|}{$\begin{array}{l}\text { Momentum flux measurement, } \mathrm{kg} / \mathrm{m}-\mathrm{sec}^{2} \\
\left(1 b_{\mathrm{m}} / \mathrm{ft}-\sec ^{2}\right)\end{array}$} \\
\hline $\begin{array}{l}\text { CCDAS } \\
\text { A.na1.og tape system }\end{array}$ & $\begin{array}{l}6793(4565) \\
7661(5148)\end{array}$ \\
\hline $\begin{array}{l}\text { Dens:ty measurpment } 961 \mathrm{~kg} / \mathrm{m}^{3}\left(601 \mathrm{~b}_{\mathrm{m}} / \mathrm{ft}^{3}\right) \text {, } \\
\mathrm{kg} / \mathrm{m}^{3}\left(1 \mathrm{~b}_{\mathrm{m}} / \mathrm{tt}^{3}\right)\end{array}$ & $12.9(0.81)$ \\
\hline
\end{tabular}


Table s. Dace presentation for ceat 100

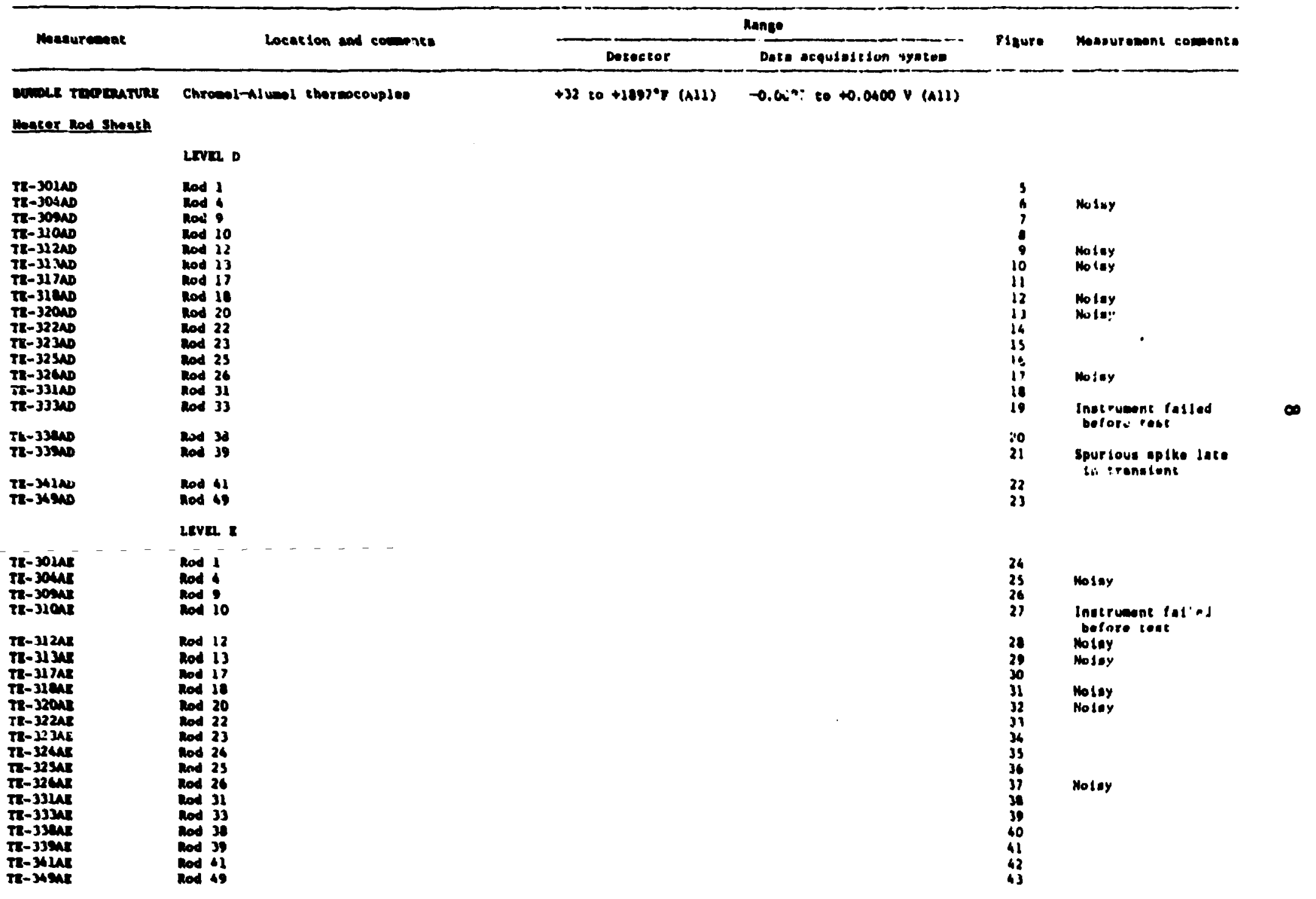




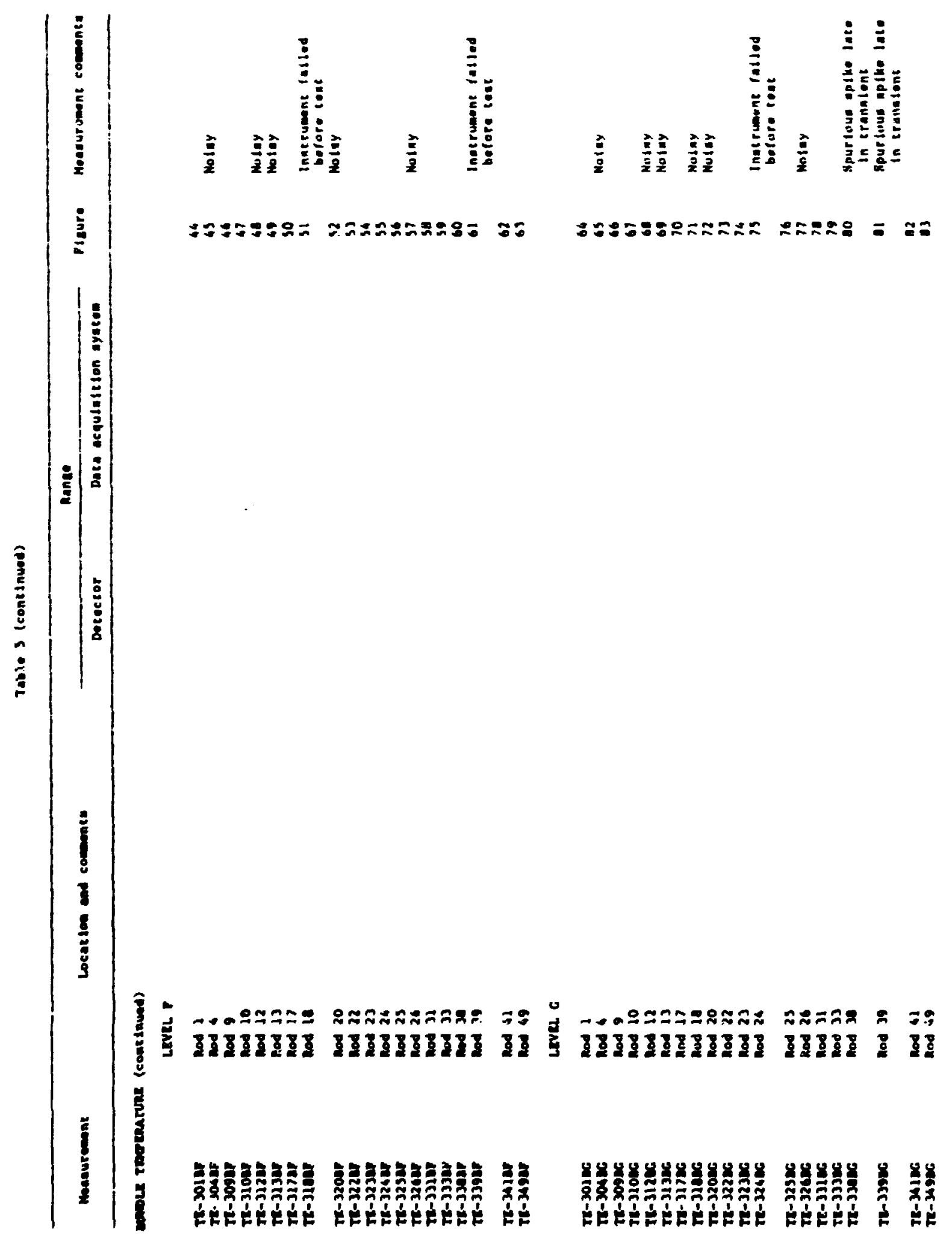




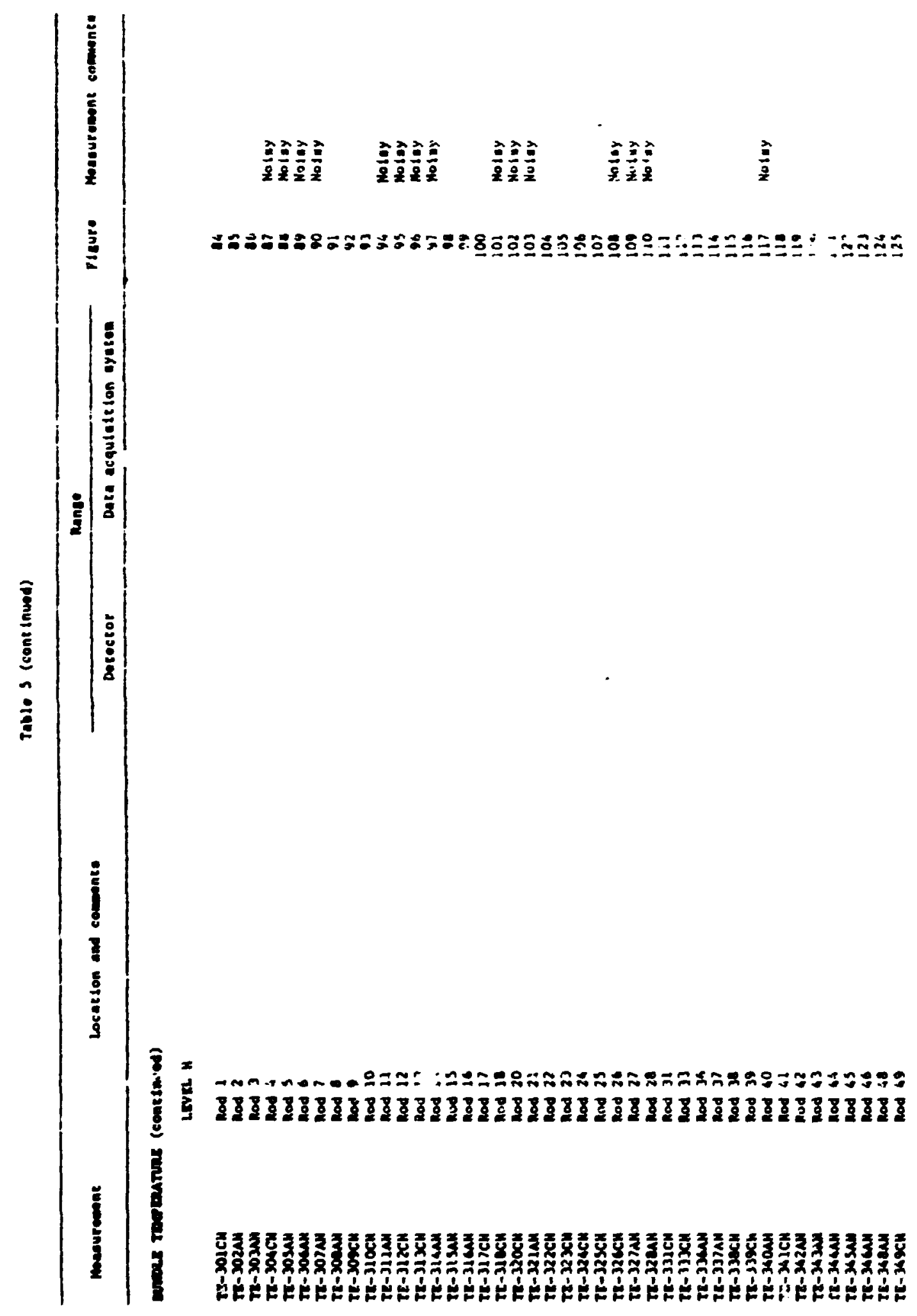


11

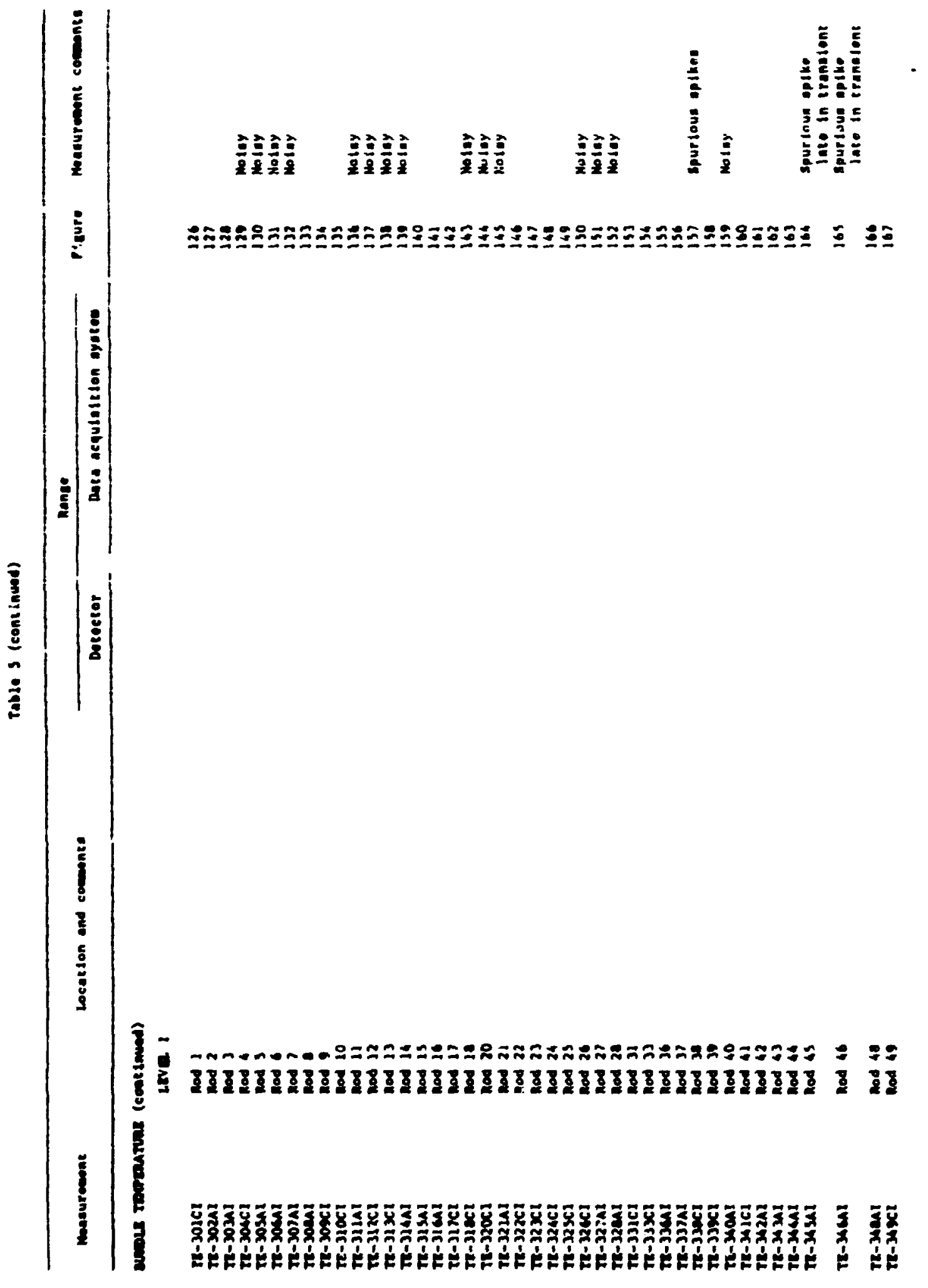




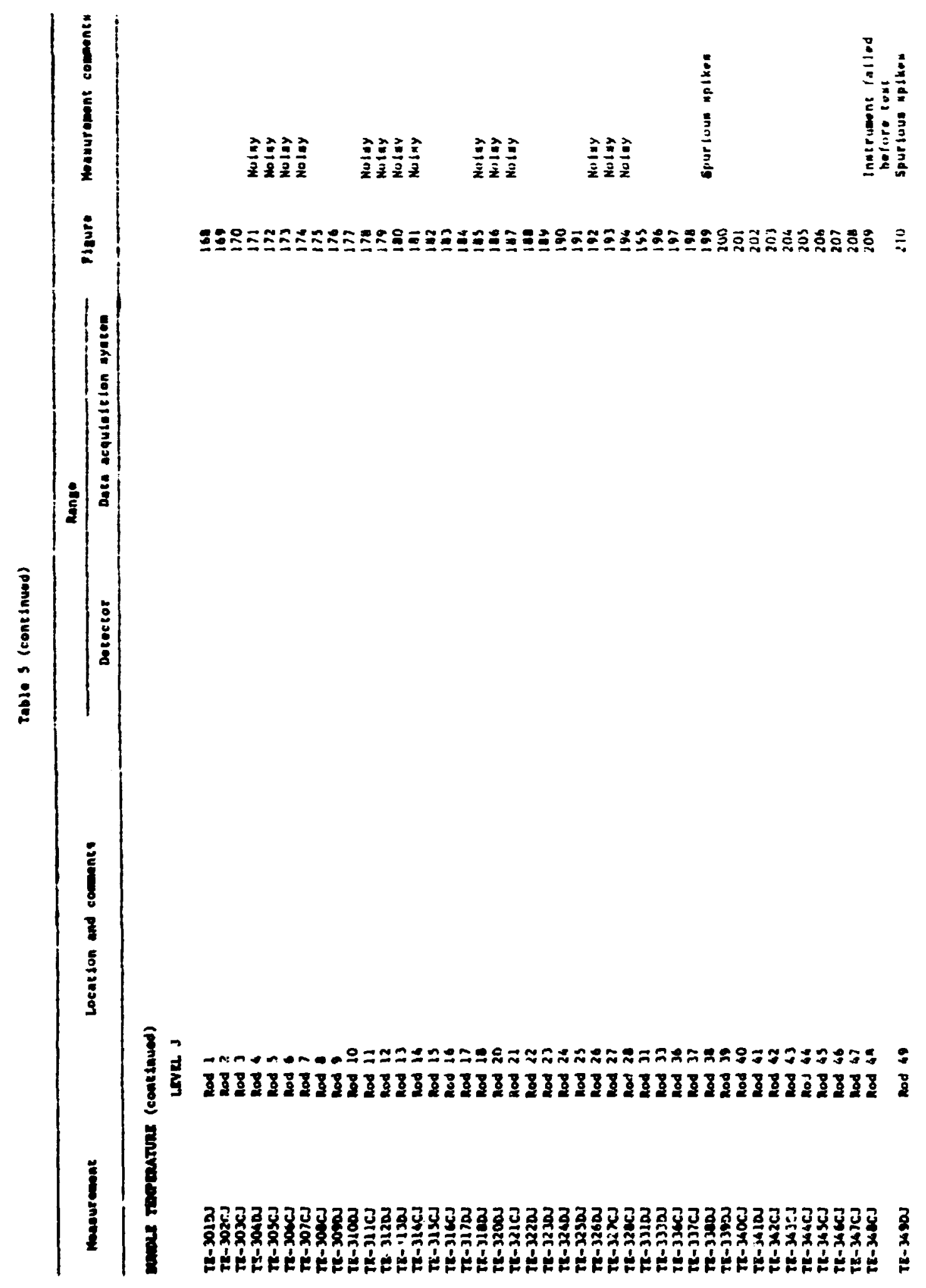


Tables (roncinued)

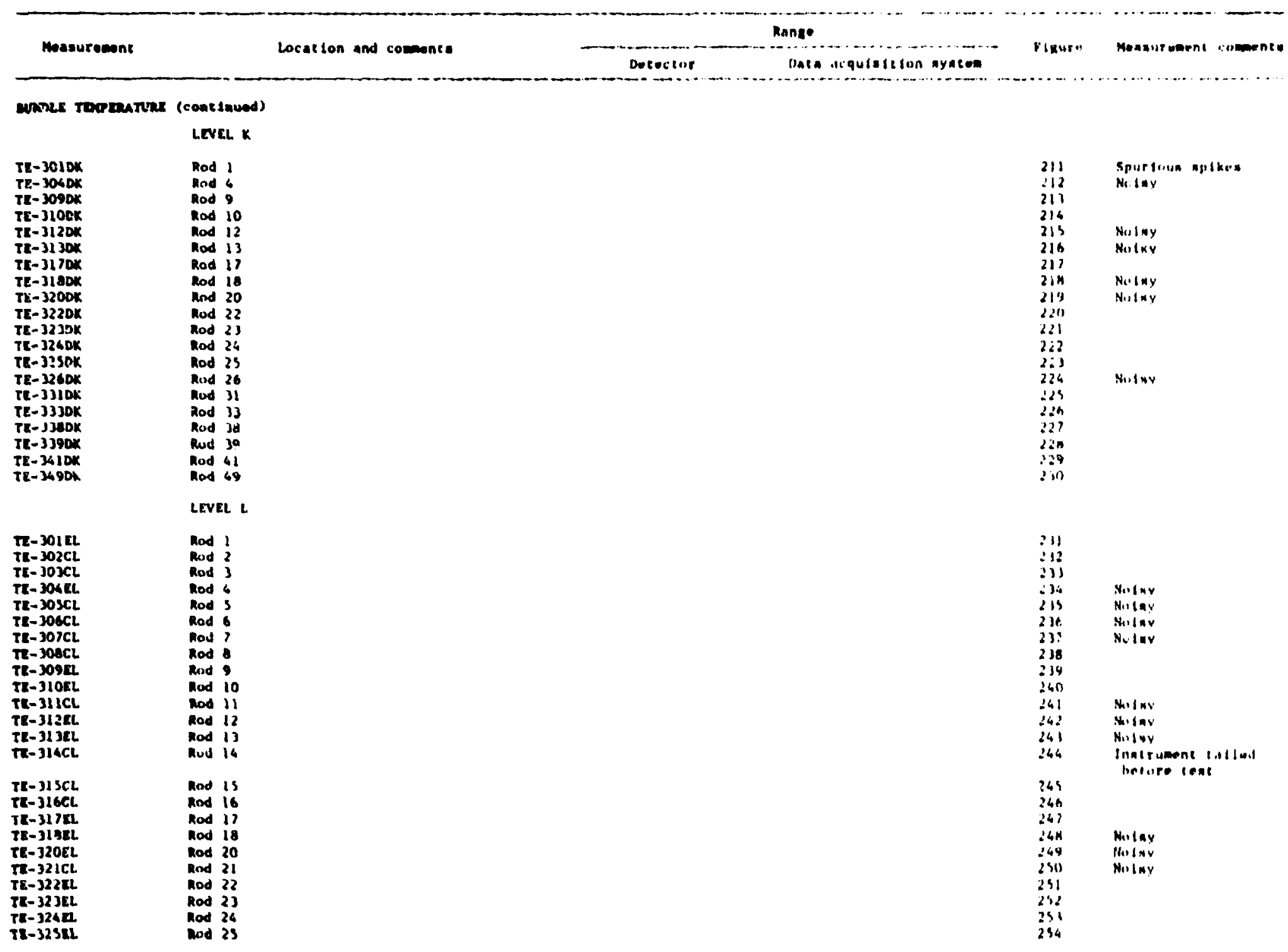




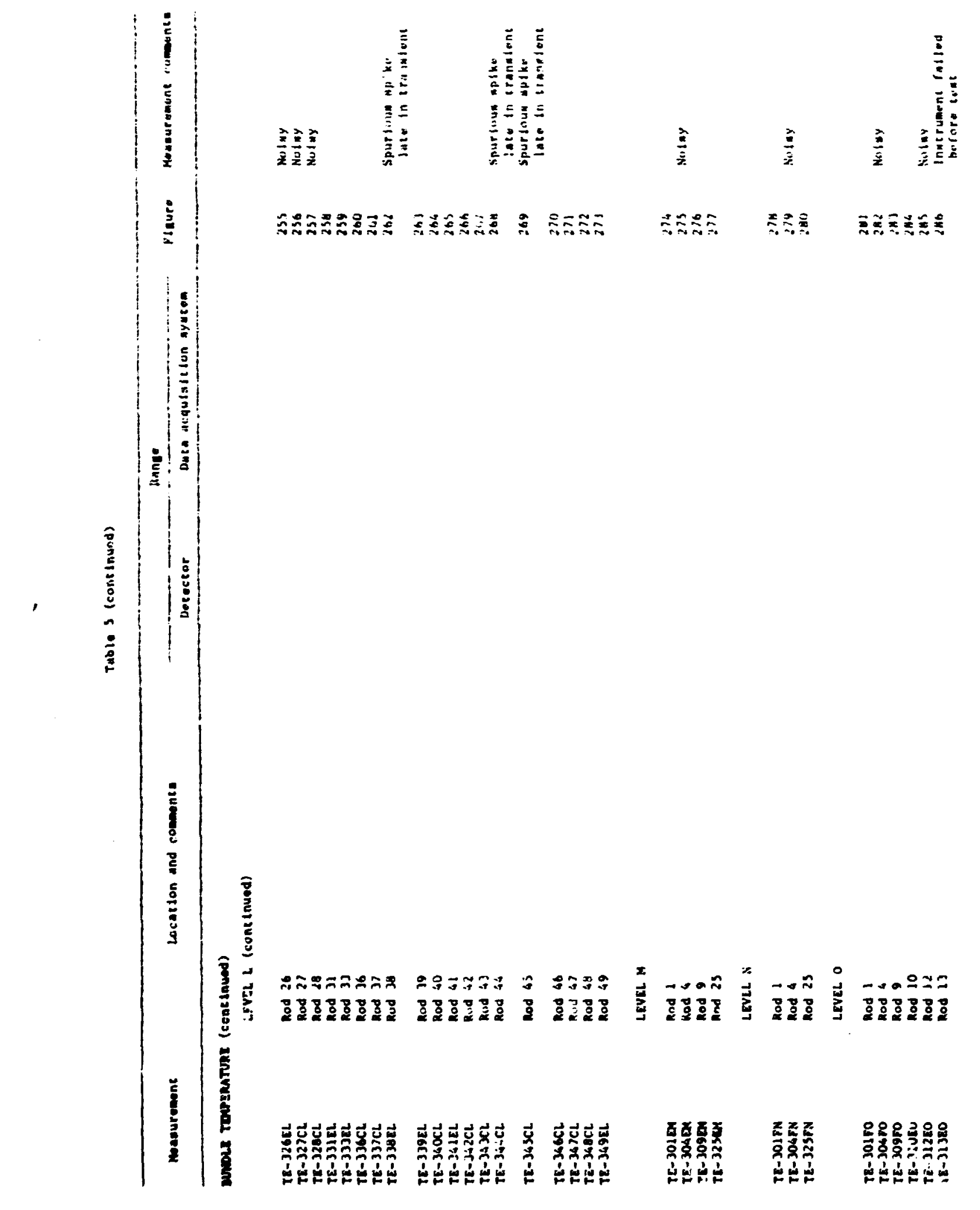


Tables (rontinued)

Measuremont Location ond comente Mange

Wrole toopanture (continuod)

LEVEL $~($ cont inued)

\begin{tabular}{|c|c|}
\hline $\begin{array}{l}T E-31750 \\
T E-31850 \\
T E-32050 \\
T E-32250 \\
T E-32350 \\
T E-32450 \\
T E-32350 \\
T E-32650 \\
T E-31150 \\
T E-33350 \\
T E-33950 \\
T E-339 E 0 \\
T E-34150 \\
T E-343 E R\end{array}$ & 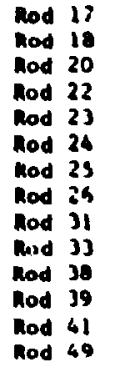 \\
\hline \multicolumn{2}{|c|}{ Mearer Rod Ceneg: } \\
\hline & LEVEL E \\
\hline TE-301NE & Rud 1 \\
\hline \multirow[t]{2}{*}{$\begin{array}{l}T E-312 n E \\
T E-322 M E\end{array}$} & $\begin{array}{l}\text { Rod } 19 \\
\text { nod } 22\end{array}$ \\
\hline & LEVEL P \\
\hline \multirow[t]{2}{*}{$\begin{array}{l}T E-301 M F \\
T I-31 \text { afr } \\
T I-322 N F\end{array}$} & $\begin{array}{l}\text { Rod } 1 \\
\text { Rod is } \\
\text { Rod } 22\end{array}$ \\
\hline & LEVEL, $c$ \\
\hline \multirow[t]{2}{*}{$\begin{array}{l}T E-301 M c \\
T E-312 \pi x \\
T E-32 \angle 1 \% \\
T E-32 \sin \end{array}$} & $\begin{array}{l}\text { Mod } 1 \\
\text { hod is } \\
\text { Rod } 22 \\
\text { Rod } 23\end{array}$ \\
\hline & LEVEL. H \\
\hline \multirow[t]{2}{*}{$\begin{array}{l}\text { TE-312004 } \\
\text { TE-3221001 }\end{array}$} & $\begin{array}{l}\text { Rod } 18 \\
\text { Rud } 22\end{array}$ \\
\hline & LEVRL J \\
\hline $\begin{array}{l}T E-301 n J \\
T E-322 \mathrm{~W}\end{array}$ & $\begin{array}{l}\operatorname{Rod} 1 \\
\operatorname{lod} 22\end{array}$ \\
\hline
\end{tabular}

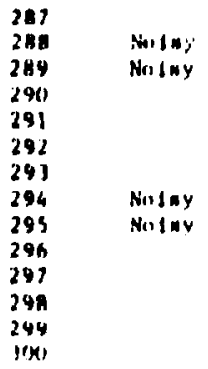

EL.

ret

18

ingy

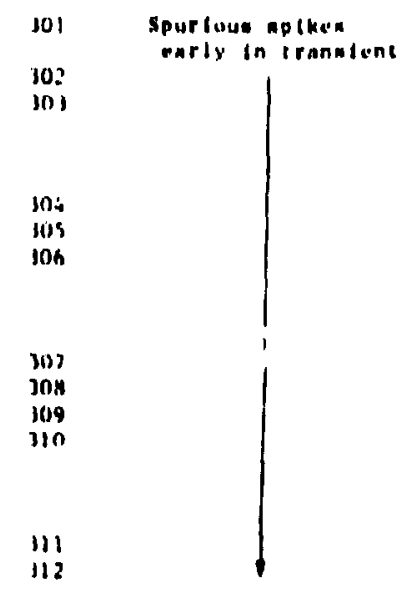

111 


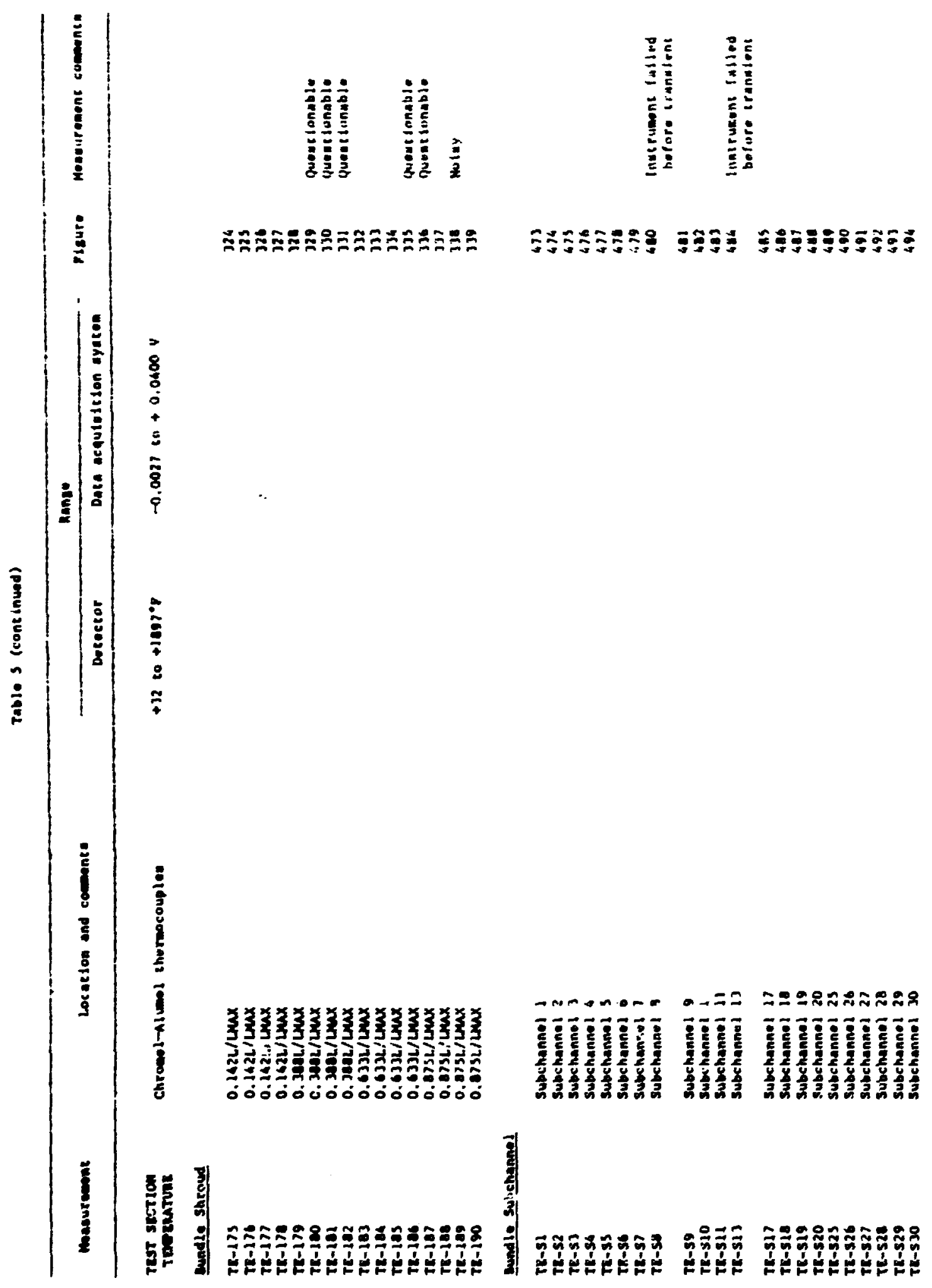




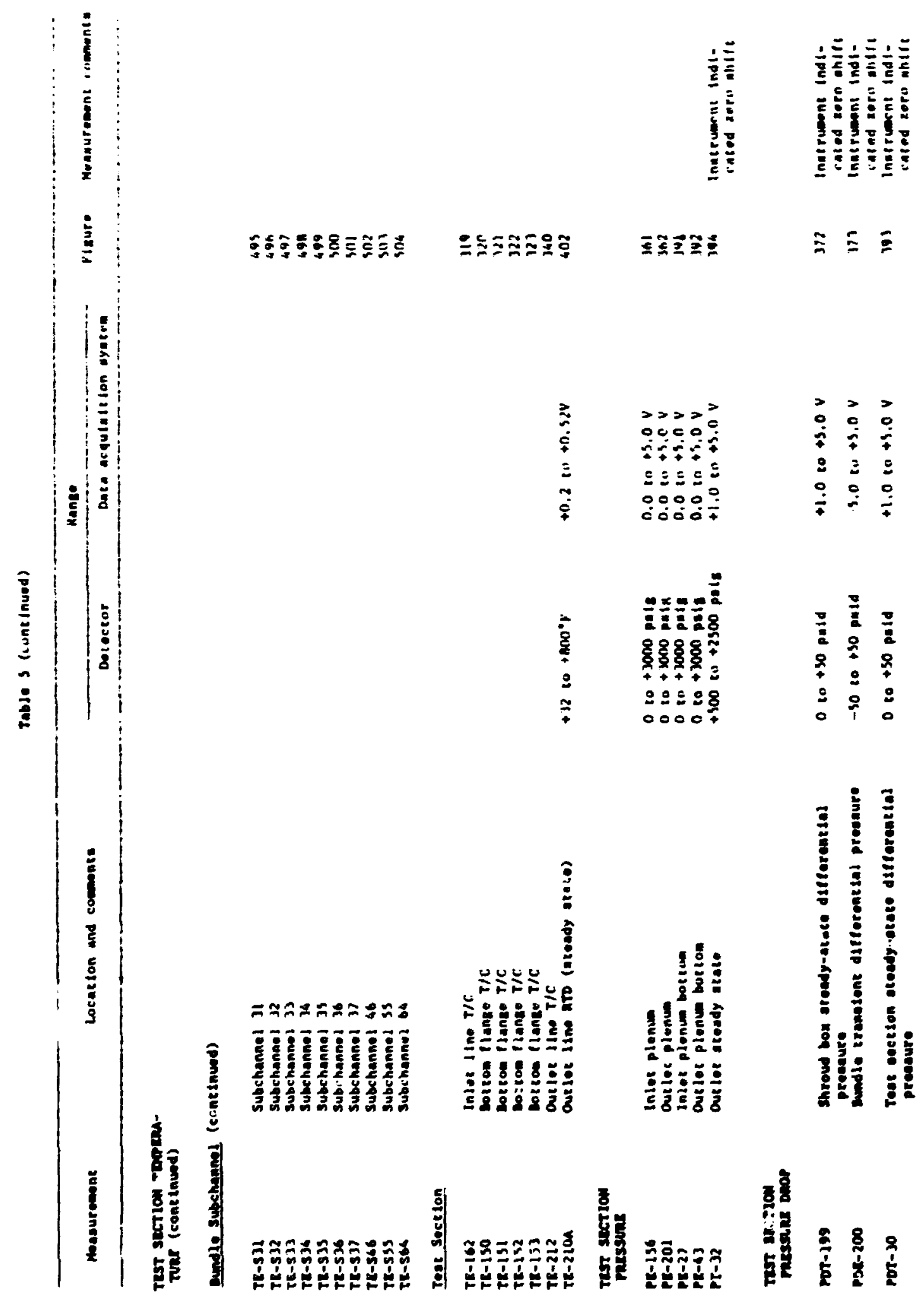


18

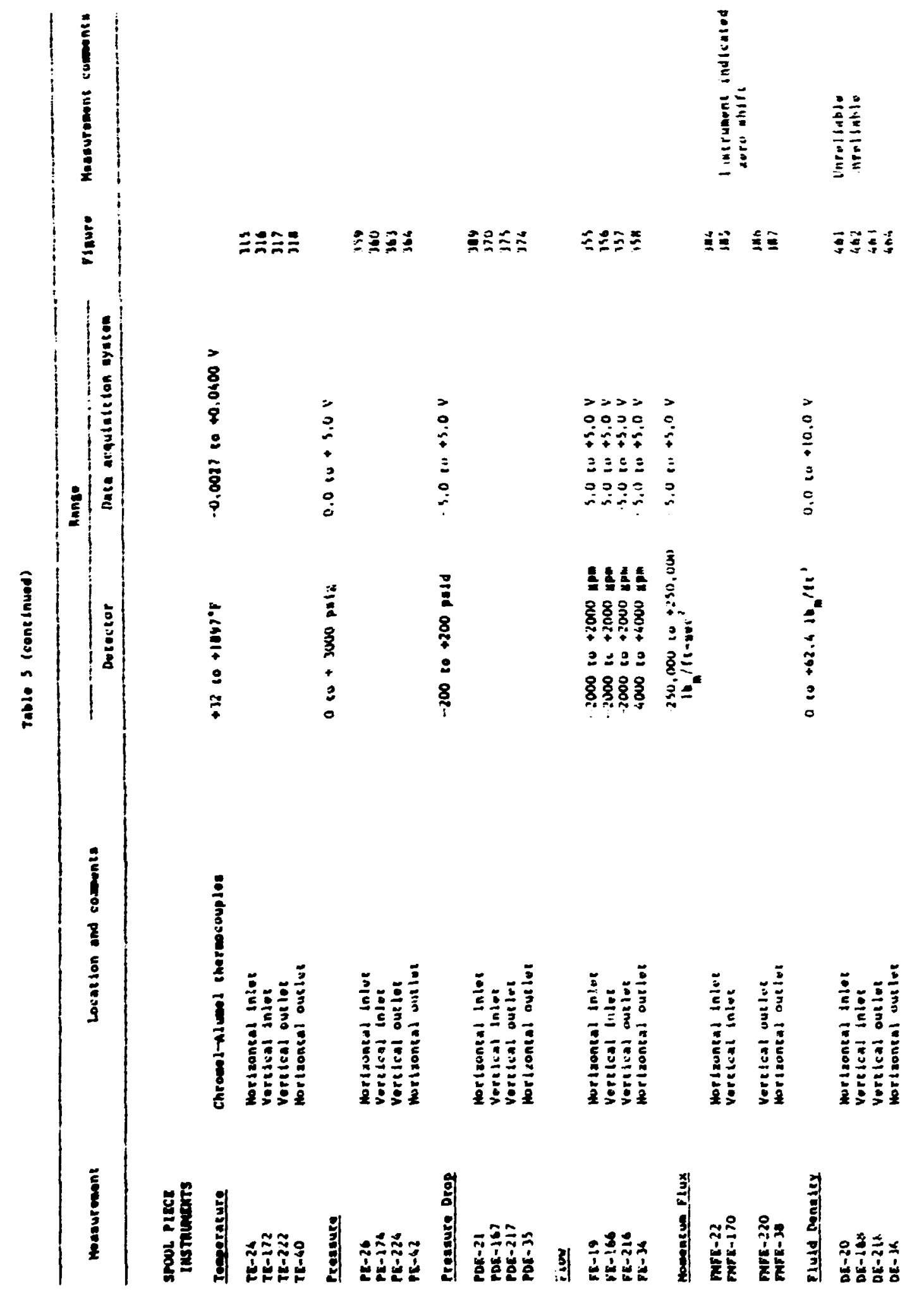


19

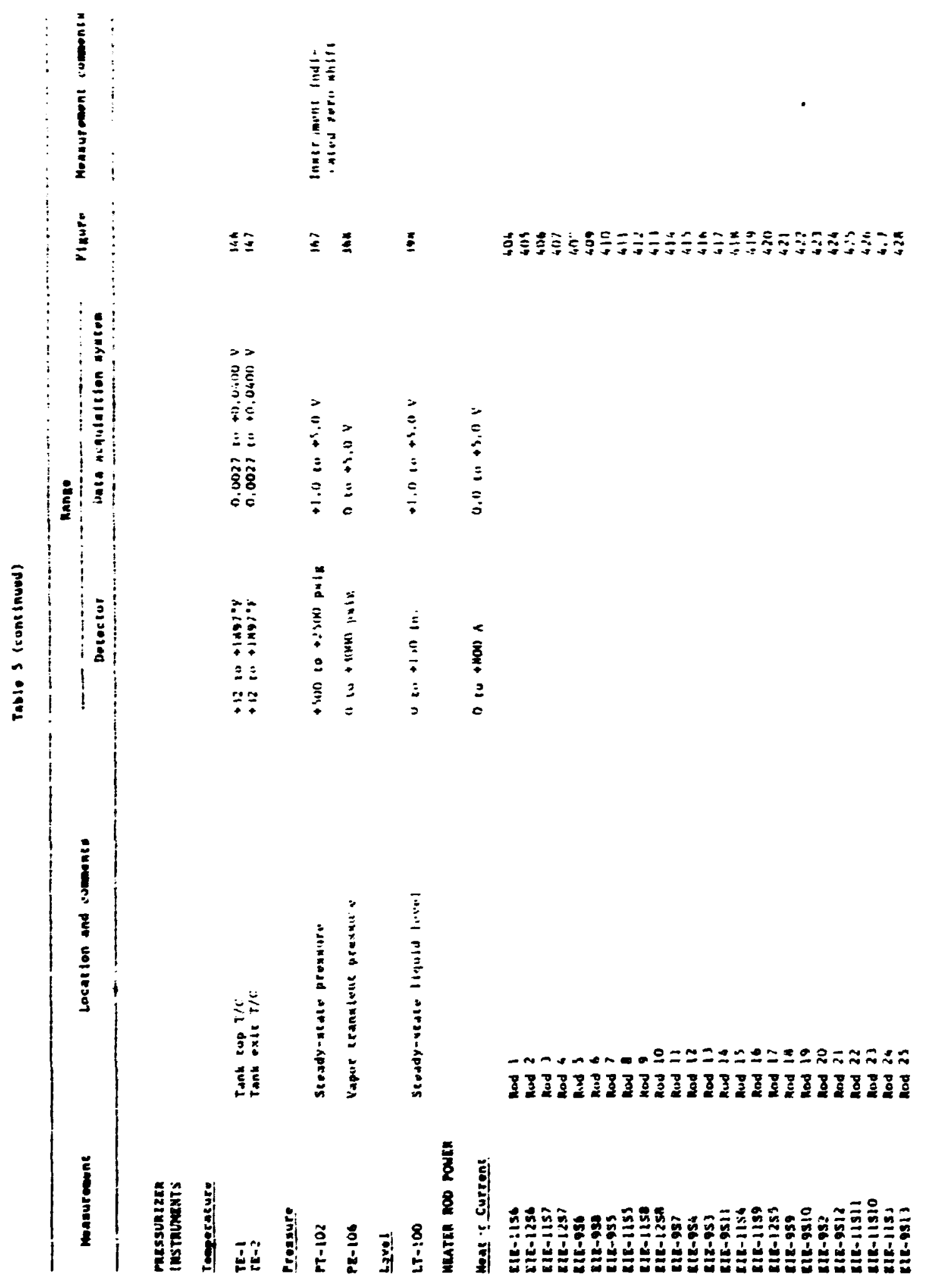


Tab10 I (continuad)

Locaurcecat

maran 200 rouna

(cont lavod)

Heater Currease (cone inumd)

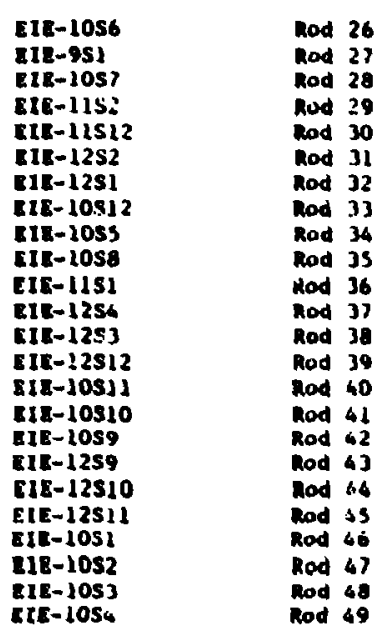

Generuton pourk:

Gomerato: Currens

EIR-9

EIE-10

EIR-11

Canereror voleare

x.25-9

ExE-10

$\operatorname{mix}-11$

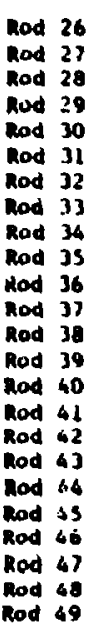

27
28

30

32

36

36

38

40

64

46

$\operatorname{lod} 49$

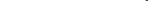


Toble 3 (eont inued)

\begin{tabular}{|c|c|c|c|c|c|}
\hline \multirow{2}{*}{ nesourenome } & \multirow{2}{*}{ Location and eomonts } & \multicolumn{2}{|r|}{ Range } & \multirow{2}{*}{ Migure } & \multirow{2}{*}{ Meneurcenent comonese } \\
\hline & & Detector & Dote aequidatition oyeten & & \\
\hline \multicolumn{6}{|l|}{ 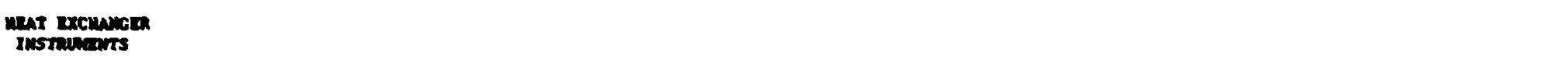 } \\
\hline Represide & Qutlet Line Mnereocouples & +32 10 +1898 & $-0.002710+0.0400 \mathrm{~V}$ & & \\
\hline 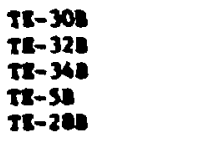 & 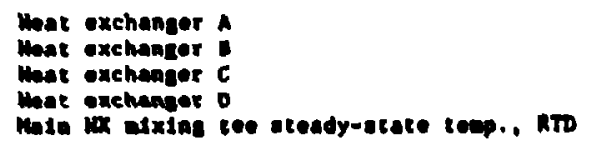 & $+3210+200^{\circ} \mathrm{r}$ & $+0.210+0.32 v$ & $\begin{array}{l}341 \\
342 \\
343 \\
349 \\
382\end{array}$ & \\
\hline secomany side & Socoodary discharge ateady-atatd texp., RTD & $+3210+500 \%$ & $+0.2 \operatorname{sen}+0.4 v$ & & \\
\hline $\begin{array}{l}\pi-523 \\
\pi-627 \\
\pi-727 \\
\pi-537\end{array}$ & 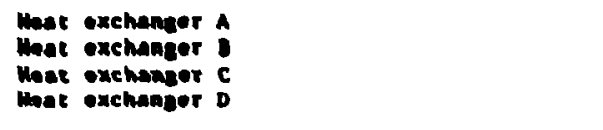 & & & $\begin{array}{l}601 \\
316 \\
316 \\
600\end{array}$ & Noiny \\
\hline \multicolumn{6}{|l|}{$\frac{\text { Eat Ixchaner }}{\text { Secondary vor }}$} \\
\hline $\begin{array}{l}n-522 \\
n-620 \\
n-720 \\
n-530\end{array}$ & 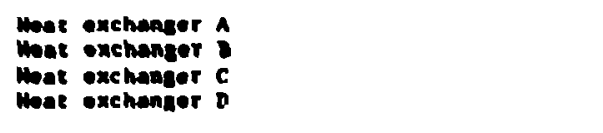 & 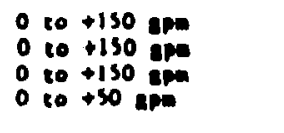 & $\begin{array}{l}+0.210+1.0 v \\
+0.210+1.0 v \\
+0.210+1.0 y \\
+0.210+1.0 v\end{array}$ & $\begin{array}{l}399 \\
331 \\
332 \\
353\end{array}$ & Nolsy \\
\hline \multicolumn{6}{|l|}{$\frac{\text { Mat Ixchanex }}{\text { Preceura }}$} \\
\hline Ple-s26 & 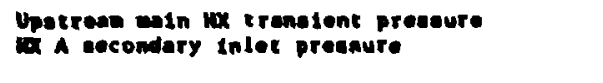 & $\begin{array}{l}0 \text { to }+3000 \text { pars } \\
0 \text { to }+350 \text { past }\end{array}$ & $\begin{array}{l}0.010+5.0 \mathrm{~V} \\
0.010+5.0 \mathrm{~V}\end{array}$ & 363 & \\
\hline \multicolumn{6}{|l|}{ Ereceaure Dres } \\
\hline $\begin{array}{l}\text { Por-49 } \\
\text { Pex }-46\end{array}$ & 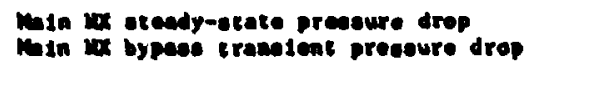 & $\begin{array}{l}0 \text { to }+24 \text { peld } \\
-200 \text { to }+200 \text { peld }\end{array}$ & $\begin{array}{l}+1.0<0+3.0 \mathrm{~V} \\
-3.0<0+3.0 \mathrm{~V}\end{array}$ & 373 & $\begin{array}{l}\text { Inetrumone anturaced } \\
\text { at } 41.0 \text { aec }\end{array}$ \\
\hline \multicolumn{6}{|c|}{$\operatorname{manar}_{\text {mins }}$} \\
\hline n-10 & Priangy etde pus tion & $080+200 \mathrm{spm}$ & $+1.0 \leqslant 0+3.0 \mathrm{~V}$ & 356 & $\begin{array}{l}\text { Inotrument coturatod } \\
\therefore 21.0 \text { ser }\end{array}$ \\
\hline PL-76 & Pup evetion tranelent precture & 0 to +3000 post & $010+5.0 \mathrm{~V}$ & 366 & \\
\hline meterb & Pritary sure iranedeat preseure drop & $-100010+1000$ pald & $-5.0<0+3.0 v$ & 378 & \\
\hline $\operatorname{se-n}$ & Pricery pue opoed & +100 to +\$600 rpa & $0+0+3.0 \mathrm{~V}$ & 395 & \\
\hline
\end{tabular}




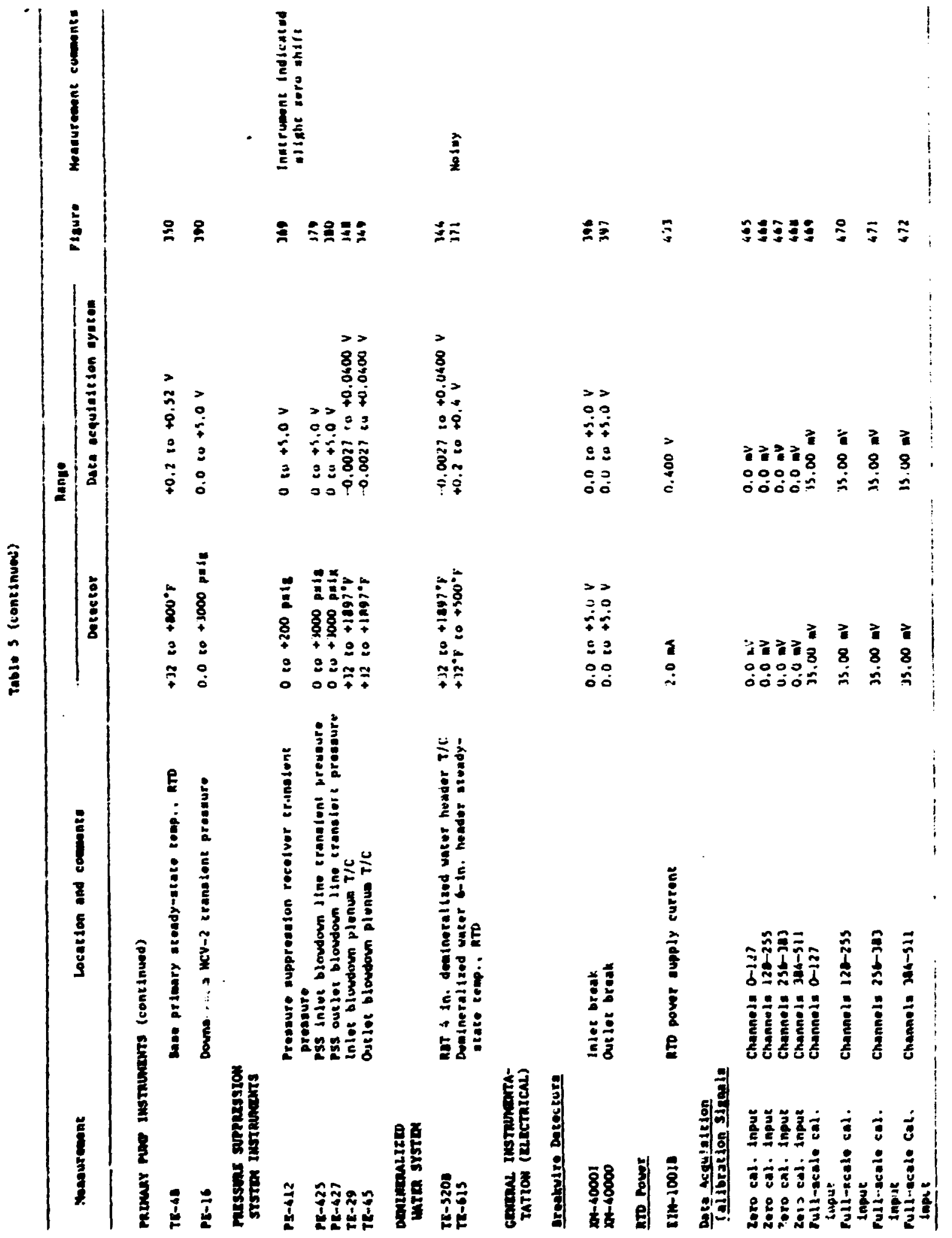




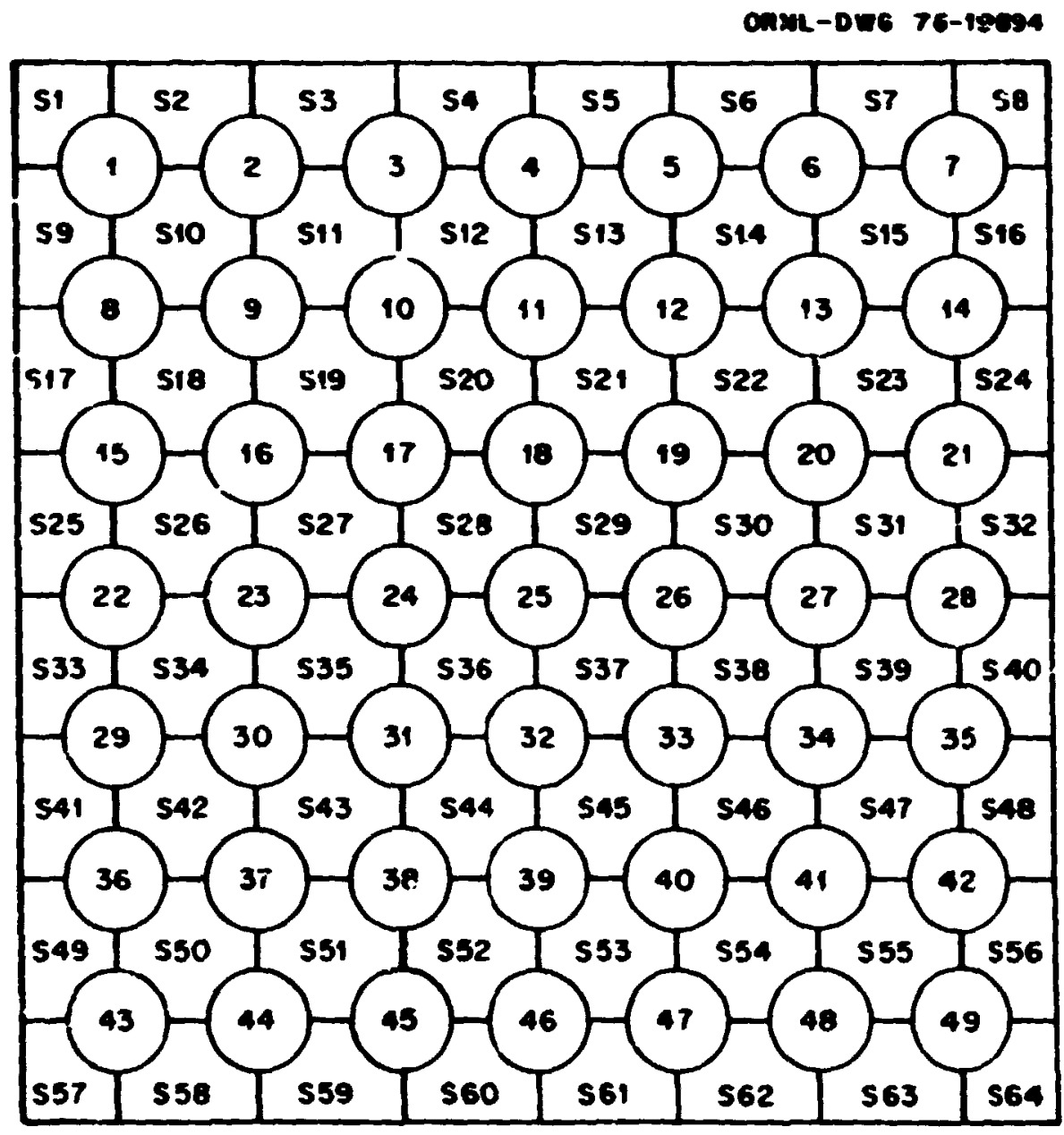

P1g. 2. Identification of THTT heiter rod and subchannel locations In bund les 1 and 2 . 


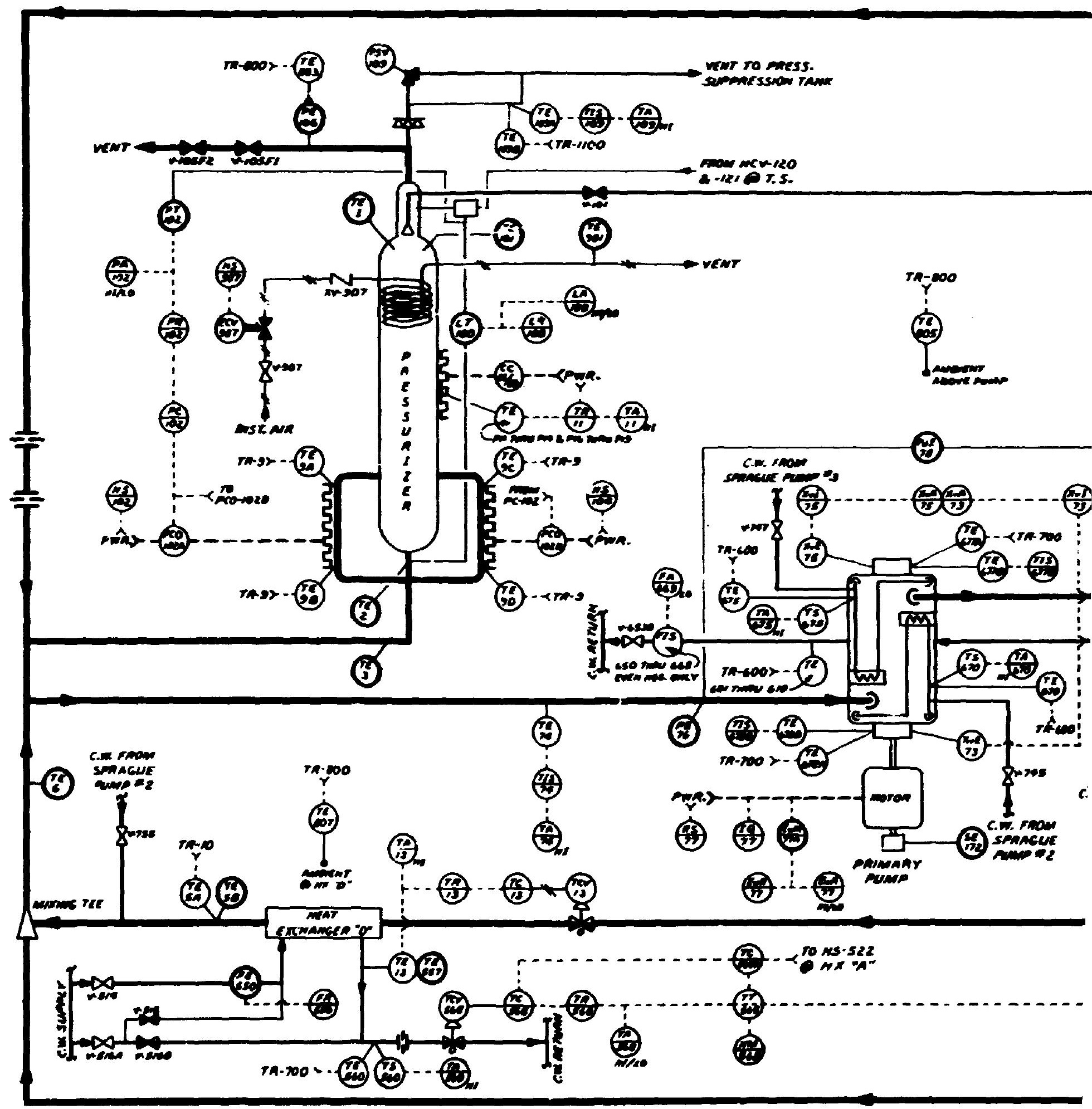

P1g. 3. THTP loop test eection detalls. 


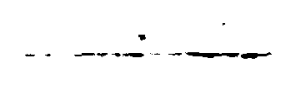

- — L

BLANK PAGE 
now 50 eness.

semesseces raner

a) $-\frac{a}{2}-\frac{a}{b}$

$=\pi, 1000$

inos mcurezo

a-12, 9 T.s.

$\rightarrow$

().

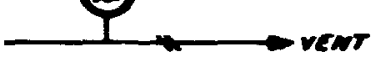

(9)

$-\infty$ p.

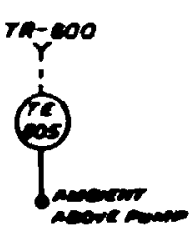

(ii) - in manmen

crang

(2)

$\Leftrightarrow$

B.

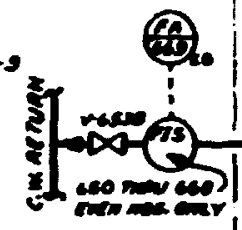

\section{vewr}<smiles>C1CCCCC1</smiles>

cmanom

0 (7)

(i)

(ऐ)

(ख)

ख....

(6)

F18. 3. TitT loop test section details.

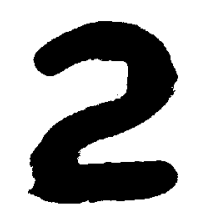




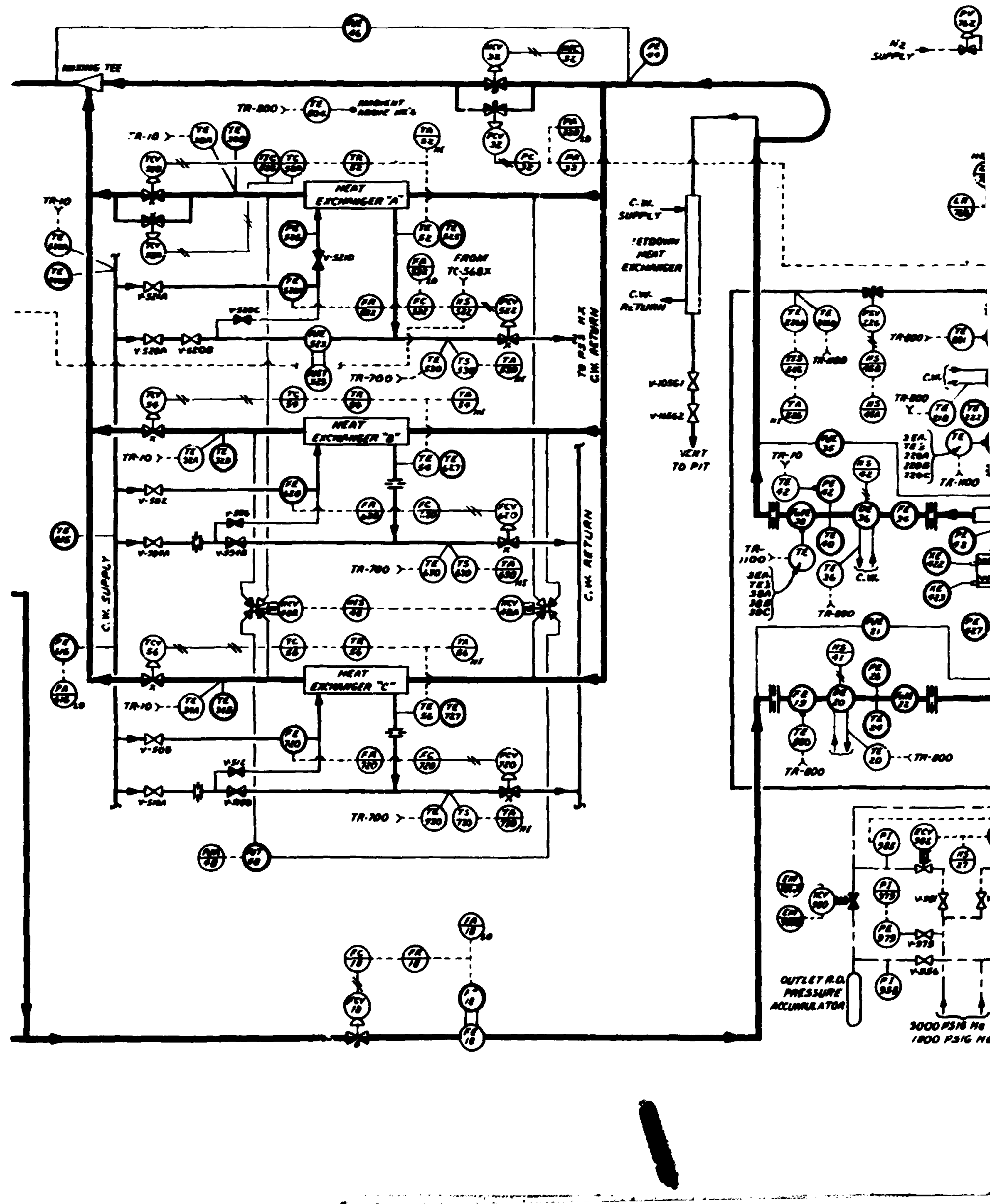


1 


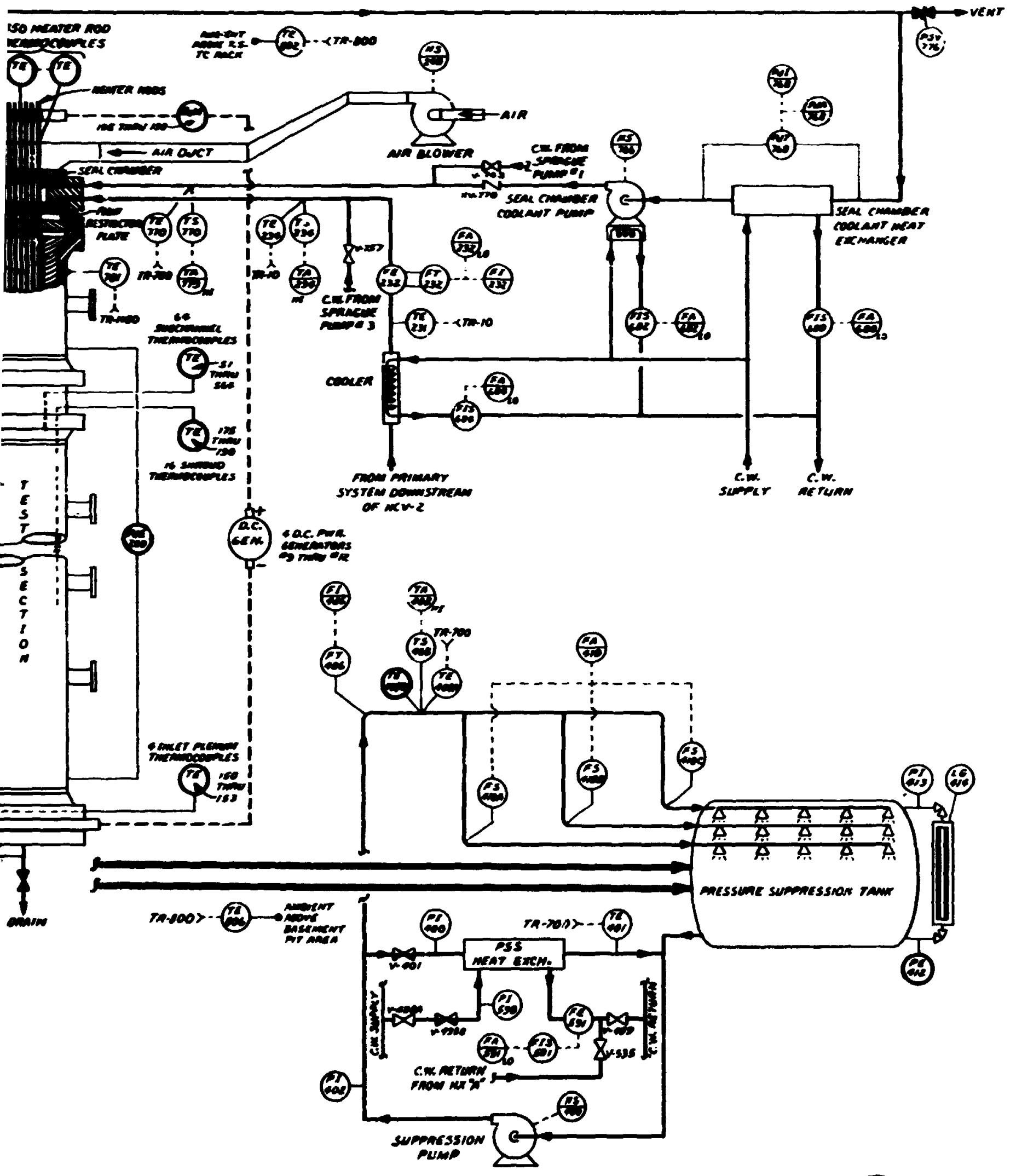




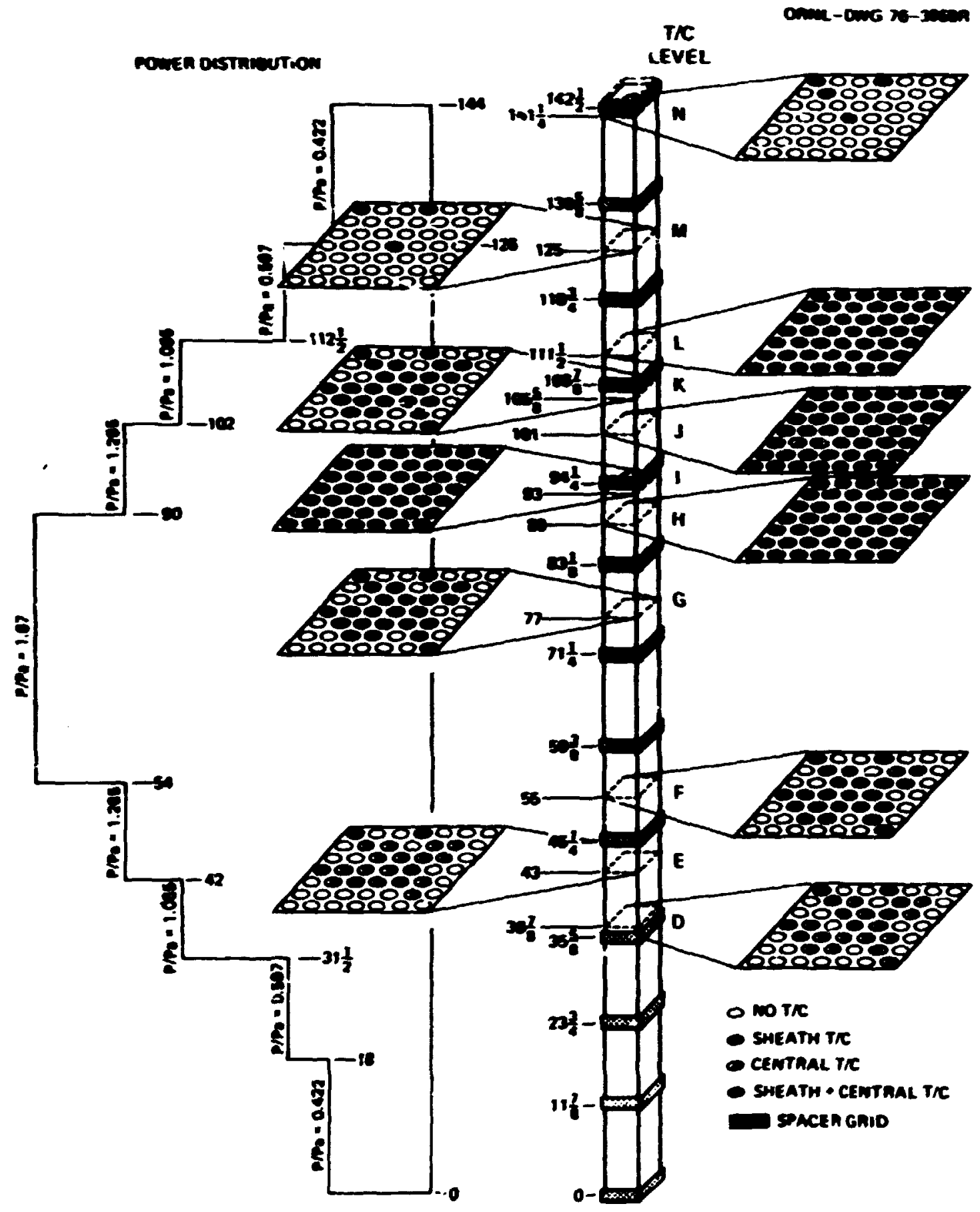

P1g. 4. Tentative location of thernocouples in THTF bundle 1 ( 1 in. = $2.54 \mathrm{cD})$. 